\title{
Market Efficiency and Real Efficiency: The Connect and Disconnect via Feedback Effects
}

\author{
Itay Goldstein and Liyan Yang*
}

January, 2014

\begin{abstract}
We study a model to explore the (dis)connect between market efficiency and real efficiency when real decision makers learn information from the market to guide their actions. We emphasize two channels that determine whether the two efficiency concepts are aligned. The "externality channel" says that individual learning outcomes may not always map into real efficiency because the presence of externality causes real decision makers to overuse the price information. The "(mis)match channel" emphasizes the fact that market efficiency concerns how much information the market reveals about the overall firm value, while improving real efficiency needs the market to reveal much information that is relevant for real decisions. Our analysis highlights the delicate link between market efficiency and real efficiency.
\end{abstract}

Keywords: Market Efficiency, Real Efficiency, Feedback Effects, Externality.

JEL Classifications: D61, D62, G14, G30

*Itay Goldstein: Department of Finance, Wharton School, University of Pennsylvania, Philadelphia, PA 19104; Email: itayg@wharton.upenn.edu; Tel: 215-746-0499. Liyan Yang: Department of Finance, Joseph L. Rotman School of Management, University of Toronto, 105 St. George Street, Toronto, Ontario M5S 3E6; Email: liyan.yang@rotman.utoronto.ca; Tel: 416-978-3930. We would like to thank Alexi Savov and the seminar participants of the 2014 ES Session on the Real Effects of Financial Markets (Philadelphia). 


\section{Introduction}

Market efficiency is a central topic in financial economics. It refers to the extent to which the prevailing market prices are informative about the future value of the traded assets. For example, Brown, Harlow, and Tinic (1988, p. 355-356) write: "the efficient market hypothesis $(\mathrm{EMH})$ claims that the price of a security at any point is a noisy estimate of the present value of the certainty equivalents of its risky future cash flows." "A market in which prices always 'fully reflect' available information is called 'efficient."' (Fama, 1970, p. 383) Due to its relation to information and prices, market efficiency is also termed as informational efficiency and price efficiency.

Regulators and academics often view promoting market efficiency as one desirable goal. For instance, O’Hara (1997, p. 270) states: "How well and how quickly a market aggregates and impounds information into the price must surely be a fundamental goal of market design." This is because informative prices are supposed to improve real decision making (such as investments). As Fama and Miller (1972, p. 335) note: "(an efficient market) has a very desirable feature. In particular, at any point in time market prices of securities provide accurate signals for resource allocation; that is, firms can make production-investment decisions...." This idea is quite natural and it goes back to Hayek (1945) who argues that the market price is an effective source of information by aggregating diverse pieces of information possessed by various market participants.

In this paper, we examine whether and when the above argument of linking market efficiency and real investment efficiency is valid in a model in which decision makers in the real side of the economy (henceforth, real decision makers) learn information from the financial market to guide their actions. In our model economy, the real decision makers are capital providers who determine how much capital to provide to a financially-constrained firm for the purpose of making real investments. ${ }^{1}$ The production technology has two features. First, there is a negative externality in capital providers' cost function when they provide

\footnotetext{
${ }^{1}$ Our model also admits an alternative macro interpretation that real decision makers are company managers in a given industry who make real investments. Their aggregate investment determines the cash flow of an asset that is an index on the industry's aggregate output. The literature has discussed other possible types of real decision makers who can learn form prices, such as regulators, product customers, input suppliers, and employees (See Bond, Edmans, and Goldstein (2012)).
} 
capital to the firm: As more capital is provided, the marginal cost of raising fund increases. We will show that this externality plays an important role in determining the link between market efficiency and real efficiency.

The second feature of the production technology is that it has two independent productivity factors - factor $\tilde{a}$ and factor $\tilde{f}$ - such as a macro factor and a firm-specific factor as in Veldkamp and Wolfers (2007), or a permanent factor and a transitory factor as in Liu, Wang, and Zha (2013). Capital providers have better private information about one factor $(\tilde{a})$ than the other $(\tilde{f})$, and hence, when offered other sources of information, they are more keen to learn about the factor $(\tilde{f})$ of which they are relatively uninformed. The other available source of information in our model is the information aggregated by the price of the firm's security traded in the financial market. That capital providers learn from prices establishes the effect that financial trading has on the real economy, and we refer to this effect as the "feedback effect."

The financial market in our economy is populated by a group of speculators who trade a security whose cash flow is correlated with the output produced by the firm. Speculators have private information about the two productivity factors $\tilde{a}$ and $\tilde{f}$. The equilibrium asset price will therefore convey information about $\tilde{a}$ and $\tilde{f}$ through their trading, although the aggregation is not perfect due to the presence of noise trading. So, capital providers can extract information about $\tilde{a}$ and $\tilde{f}$ from prices to guide their decisions on real investments.

Our analysis highlights two channels, which we label as the "externality channel" and the "(mis)match channel," respectively, that are important in determining the link between market efficiency and real efficiency. The thought experiment goes as follows. Suppose, for example, that some exogenous reasons (such as changes in regulation rules or informationacquisition technology) cause the trading environment to change such that market efficiency gets improved. We care about whether real efficiency also improves, so that market efficiency is a good proxy for real efficiency. The externality channel concerns whether individual learning can be efficiently aggregated: Assuming that each capital provider can learn more information from the more informationally-efficient price after the trading environment changes, does this improved individual learning outcome readily translate into a higher level of real efficiency in aggregate? 
We find that the answer to the above question is positive if and only if there is not much externality in capital providers' cost function. If each individual capital provider learns more information from the price, two counteracting effects on the real efficiency arise. The positive effect is straightforward: After equipped with better information, each capital provider's investment decision improves, which tends to improve the aggregate investment efficiency. However, the presence of a negative externality also gives rise to a negative effect - i.e., the "inefficient" use of information. As compared to what is socially optimal, capital providers underuse their private information and overuse the common price information, because the common price information helps them to better predict the aggregate investment that affects their future payoff through the externality. The more accurate is the price information, the larger is the efficiency loss induced by this bias. When the externality is strong, this negative effect can dominate so that improved individual learning can actually harm real efficiency in aggregate. We present this result associated with the externality channel in Section 5 .

The (mis)match channel emphasizes a more basic fact - market efficiency and real efficiency are simply two different concepts. Market efficiency concerns the price informativeness about the future value of the traded asset. Real efficiency is about the efficiency of resource allocation, and it can be improved only if the price reveals information that real decision makers (capital providers in our model) care to learn. In the context of our model, capital providers wish to learn factor $\tilde{f}$ more than factor $\tilde{a}$. Thus, if the improved market efficiency in the thought experiment is driven by information about $\tilde{f}$, then real efficiency also improves (in the absence of externality). By contrast, if the improved market efficiency is driven by information about $\tilde{a}$, then real efficiency can be reduced. In other words, whether market efficiency and real efficiency are aligned depends on whether the information revealed by the price matches or mismatches the information that capital providers want to learn. We present this result related to this (mis)match channel in Section 6.

Dow and Gorton (1997) have also theoretically studied the link between market efficiency and real efficiency. They identify two mechanisms for informationally-efficient price to enhance economic efficiency, namely that managers learn from the market and that the market motivates managers to produce information in the presence of agency issues. They rely on multiple equilibria to break the link between market efficiency and real efficiency - in one 
equilibrium, speculators produce information and managers base investment decisions on prices, while in the other equilibrium, no information is produced and no investment is made because the project has a negative NPV ex ante; however, in the second equilibrium, real efficiency is low although the price is informationally efficient. By contrast, in our model, we rely solely on the learning mechanism to link market efficiency and real efficiency, and when we study the possible delink between these two efficiency concepts, we identify two new channels, namely the externality channel and the (mis)match channel. So, our study is different from and thus complements Dow and Gorton (1997).

The interesting models in Subrahmanyam and Titman $(2001,2013)$ feature feedback effects and externality as well. In Subrahmanyam and Titman (2001), there is interdependence among the payoffs of a firm's non-financial stakeholders, such as customers and employees, and this feature generates cascades, wherein relatively small price moves trigger substantial changes in fundamentals. In Subrahmanyam and Titman (2013), two private firms learn from the asset price of a traded public firm and the investments of the two private firms exert externality on each other, which in equilibrium generates a weak correlations between stock prices and cash flows of the public firm as well as a positive correlation between stock prices and aggregate outputs. In contrast, the externality in our model occurs among the investments of the traded firm and our focus is on the link between the market efficiency and real efficiency concepts. Also, the players involved with externality in Subrahmanyam and Titman $(2001,2013)$ do not have private information, and thus the inefficient use of price vs. private information, which is key to our externality channel, is absent in their analyses.

Broadly, our study is most closely related to two strands of literature: the economics literature on the use of public vs. private information, and the finance literature on the informational feedback from asset prices to real decisions. These two literatures have developed independently, by and large. By combining the elements separately studied in both literatures in a unifying framework, our paper offers new insights on the link between market efficiency and real efficiency. Our analysis suggests that although providing traders with more information tends to improve market efficiency, it may reduce real efficiency, depending on the type of information being added and the interaction among real decision makers.

Morris and Shin (2002) kicked off the debate on whether public information has been 
used too much by showing that public information may have a detrimental welfare effect in a beauty-contests economy where coordination is socially wasteful. In contrast, Angeletos and Pavan (2004) and Hellwig (2005) show that public information can be welfare improving in economies with payoff externalities, and Cornand and Heinemann (2008) show that public information may have a positive welfare effect when only a fraction of market participants can see it. Angeletos and Pavan (2007) have considered a large class of Gaussian-quadratic economies featuring externalities to study the efficient use of public and private information. In a monetary economy, Amador and Weill (2010) find that more precise public information can reduce welfare through inducing agents to rely less on their exogenous private information. Colombo, Femminis, and Pavan (2013) study the link between efficient use of information and efficient production of information in economies featuring externalities. Our paper intersects with and complements this literature through the "externality channel," wherein the endogenous price information serves as a public signal to capital providers who overuse price information relative to the first-best investment policy.

The literature on the real effect of financial markets is both empirical and theoretical. ${ }^{2}$ The empirical studies document that stock prices indeed contain valuable information relevant to real decisions and that decision makers appear to learn information from prices (e.g., Luo, 2005; Chen, Goldstein, and Jiang, 2007; Edmans, Goldstein, and Jiang, 2012; Foucault and Frésard, 2013). The theoretical studies in this literature find that seriously modelling the real effect of markets often helps to explain a range of phenomena that otherwise appear puzzling, such as manipulative short selling (Goldstein and Guembel, 2008), information-based trading (Bond and Eraslan, 2010), cross-listing (Foucault and Gehrig, 2008), commodity market fluctuations (Sockin and Wei, 2013), excess stock return volatility (Ozdenoren and Yuan, 2008), and the value of noncontrolling blockholders (Edmans, 2009; Goldman and Strobl, 2013). Our study complements this literature by characterizing conditions under which market efficiency and real efficiency are (mis)aligned and by providing a unifying framework of analyzing efficient use of price information and private information.

\footnotetext{
${ }^{2}$ See Bond, Edmans, and Goldstein (2012) for a recent survey.
} 


\section{The Model}

\subsection{Environment}

We consider an extension of the model in Goldstein, Ozdenoren, and Yuan (2013). There are two types of risk-neutral players - a continuum $[0,1]$ of "speculators" (labeled by $i$ ) who trade one risky asset and a continuum $[0,1]$ of "capital providers" (labeled by $j$ ) whose actions jointly determine the asset's cash flow. The risky asset can be interpreted as a stock of a firm which is financially constrained and needs capital from outside capital providers to make real investments. Alternatively, the risky asset can be interpreted as an index on the aggregate stock market; capital providers in this case are the managers of those companies included in the index and their real investment decisions determine the cash flow on the index. Capital providers make the real investment decisions under both interpretations. To ease exposition, we stick to the first micro interpretation in setting up the model, although we frequently explain how to understand our model in a macro setting, too.

As will become clear later, assuming a continuum of speculators who are endowed with diverse signals captures the idea that the financial market aggregates value-relevant information inherently dispersed among market participants and therefore provides useful informa-

tion for real decision makers. Assuming a continuum of capital providers who are endowed with diverse signals enables us to implement our first channel, the "externality channel," of examining the link between market efficiency and real efficiency - namely that the accuracy of the information in the market price may not always be readily translated into real efficiency, because the price information also affects the coordination motives among capital providers when they make real decisions.

There are three dates, $t=0,1,2$. At date 0 , speculators trade in the asset market based on their private information about productivity factors related to the asset's future cash flows, and the equilibrium asset price aggregates their private information. At date 1, after observing the asset price and receiving private information, capital providers decide how much capital they provide to the firm, and the firm undertakes investment accordingly. At date 2 , the cash flow is realized, and all agents get paid and consume. 


\subsection{Investment Technology}

The firm in our economy has access to the following production technology:

$$
q\left(k_{j}\right)=\tilde{A} \tilde{F} k_{j},
$$

where $k_{j}$ is the amount of capital that the firm raises from capital provider $j$ at date $1, q\left(k_{j}\right)$ is the date- 2 output that is generated by the investment $k_{j}$, and $\tilde{A} \geq 0$ and $\tilde{F} \geq 0$ are two productivity factors. Let $\tilde{a}$ and $\tilde{f}$ denote the natural $\operatorname{logs}$ of $\tilde{A}$ and $\tilde{F}$, i.e., $\tilde{a} \equiv \log \tilde{A}$ and $\tilde{f} \equiv \log \tilde{F}$. We assume that $\tilde{a}$ and $\tilde{f}$ are normally distributed:

$$
\tilde{a} \sim N\left(0,1 / \tau_{a}\right) \text { and } \tilde{f} \sim N\left(0,1 / \tau_{f}\right),
$$

where $\tau_{a}>0, \tau_{f}>0$, and $\tilde{a}$ and $\tilde{f}$ are mutually independent. ${ }^{3}$

Factors $\tilde{A}$ and $\tilde{F}$ represent two dimensions of uncertainty that affect the cash flow of the traded firm. ${ }^{4}$ For example, one dimension can be a factor related to the asset in place, the other one can be the demand for the firm's future products, and the overall future sales are jointly determined by these two factors. Also, $\tilde{A}$ can be thought of as an aggregate macro factor and $\tilde{F}$ can be thought of as a firm-specific factor (Veldkamp and Wolfers, 2007). If one interprets our model as a macro setting where the traded asset is an index and capital providers are each composing firm, the factor $\tilde{A}$ can be thought of as the permanent component in the total productivity and the factor $\tilde{f}$ is the transitory component, as in Liu, Wang, and Zha (2013, p. 1154-1155). The two-factor structure is a parsimonious modeling device for our second channel, the "(mis)match channel," of examining the link between market efficiency and real efficiency - i.e., the factor that capital providers want to learn the most can be very different from the factor of which the price is most informative. In Section 6.3, we will show that this feature is important for generating our results.

At date $t=1$, each capital provider $j$ chooses the level of capital (and hence investment) $k_{j}$. Providing capital incurs a private cost according to the following functional form:

$$
c\left(k_{j} ; K\right)=\frac{1}{2} c_{0} k_{j}^{2} K^{\gamma},
$$

\footnotetext{
${ }^{3}$ The assumption that $\tilde{a}$ and $\tilde{f}$ have a mean of 0 is without loss of generality. Assuming non-zero means is equivalent to renormalizing the cost parameter $c_{0}$ introduced shortly.

${ }^{4}$ Several papers in the finance literature have also specified that the value of the traded security is affected by more than one fundamental; e.g., Froot, Scharfstein, and Stein (1992), Goldman (2005), Goldstein and Yang (2012), and Kondor (2012), among others.
} 
where $c_{0}>0$ and $\gamma>0$ are constant and $K \equiv \int_{0}^{1} k_{j} d j$ is the (equilibrium) aggregate investment level. The cost can be the monetary cost of raising the capital or the effort incurred in monitoring the investment. The parameter $c_{0}$ controls the size of the cost relative to the output $q\left(k_{j}\right)$. As in in Goldstein, Ozdenoren, and Yuan (2013), we assume that the cost $c\left(k_{j} ; K\right)$ is increasing and convex in capital provider $j$ 's own investment $k_{j}$.

Unlike Goldstein, Ozdenoren, and Yuan (2013), we here also assume that investments generates externality, so that capital provider $j$ 's cost positively depends on the aggregate investment level $K$, where parameter $\gamma$ determines the level of externality. This externality is particularly reasonable when our model is interpreted as a macro setting in which the asset is an index on the aggregate stock market or on a particular industry. For example, the aggregate capital inflow to the particular industry may drive up the interest rate, which in turn raises each capital provider's financing cost. Or more generally, the production may be involved with a particular input (money can be one input, and so is specialized labor), and the aggregate investment can push up the price for that input and hence the cost of each investment. We will show (in Proposition 3) that the externality level $\gamma$ is crucial in determining whether the accuracy of the information in the market price can be translated into real efficiency (and hence the name of the "externality channel").

We also follow Goldstein, Ozdenoren, and Yuan (2013) and assume that each capital provider $j$ captures proportion $\beta \in(0,1)$ of the full output $q\left(k_{j}\right)$ by providing $k_{j}$, and thus his payoff from the investment is $\beta q\left(k_{j}\right)$. Capital provider $j$ chooses $k_{j}$ to maximize the payoff $\beta q\left(k_{j}\right)$ he captures from the firm minus his cost $c\left(k_{j} ; K\right)$ of raising capital, conditional on his information set, $\mathcal{I}_{j}$, at date $t=1$. That is, capital provider $j$ chooses $k_{j}$ to solve

$$
\max _{k_{j}} E\left[\beta \tilde{A} \tilde{F} k_{j}-\frac{1}{2} c_{0} k_{j}^{2} K^{\gamma} \mid \mathcal{I}_{j}\right] .
$$

where he as an atomistic player takes $K$ as given.

\subsection{Information Structure}

Capital provider $j$ 's information set $\mathcal{I}_{j}$ consists of the asset price $\tilde{P}$ formed at date 0 , and

two additional private signals regarding productivity factors $\tilde{a}$ and $\tilde{f}$. Specifically, we assume 
that he perfectly observes $\tilde{a}$ but observes a noisy signal about $\tilde{f}$ :

$$
\tilde{s}_{j}=\tilde{f}+\tilde{\varepsilon}_{s, j},
$$

where $\tilde{\varepsilon}_{s, j} \sim N\left(0,1 / \tau_{s}\right)$ (with $\left.\tau_{s}>0\right)$ and $\tilde{\varepsilon}_{s, j}$ is independent of $\tilde{f}$ and $\tilde{a}$. That is, $\mathcal{I}_{j}=$ $\left\{\tilde{a}, \tilde{s}_{j}, \tilde{P}\right\}$. So, capital providers care to learn information about $\tilde{f}$ since he has already known $\tilde{a}$. This is an extreme version that different factors can be exposed to asymmetric information in different degrees (among capital providers and speculators), which creates the scope for the discrepancy between what information capital providers want to learn and what information the price reveals (and hence the name of "(mis)match channel"). In Section 7, we will extend our model to equip capital providers with noisy signals about $\tilde{a}$ as well, and show that our results go through as long as the signal quality about $\tilde{a}$ and the signal quality about $\tilde{f}$ are sufficiently different.

Each speculator $i$ is endowed with two noisy signals about $\tilde{a}$ and $\tilde{f}$, respectively:

$$
\tilde{x}_{i}=\tilde{a}+\tilde{\varepsilon}_{x, i} \text { and } \tilde{y}_{i}=\tilde{f}+\tilde{\varepsilon}_{y, i},
$$

where $\tilde{\varepsilon}_{x, i} \sim N\left(0,1 / \tau_{x}\right)$ (with $\left.\tau_{x}>0\right), \tilde{\varepsilon}_{y, i} \sim N\left(0,1 / \tau_{y}\right)$ (with $\left.\tau_{y}>0\right)$, and they are

mutually independent of $\{\tilde{a}, \tilde{f}\}$. That is, speculator $i$ 's information set is $\mathcal{I}_{i}=\left\{\tilde{x}_{i}, \tilde{y}_{i}\right\}$. The market price $\tilde{P}$ will aggregate their signals $\left\{\tilde{x}_{i}, \tilde{y}_{i}\right\}$ through their trading in the financial market, and hence $\tilde{P}$ will contain information about $\tilde{a}$ and $\tilde{f}$, which is useful for capital providers to make real investment decisions. We next elaborate on the formation of prices.

\subsection{Trading and Price Formation}

At date $t=0$, speculators submit market orders as in Kyle (1985) to trade the risky asset in the financial market. They can buy or sell up to a unit of the risky asset, and thus speculator $i$ 's demand for the asset is $d(i) \in[-1,1]$. This position limit can be justified by borrowing/short-sales constraints faced by speculators. As argued by Goldstein, Ozdenoren, and Yuan (2013), the specific size of this position limit is not crucial, and what is crucial is that speculators cannot take unlimited positions. Speculators are risk neutral, and therefore they will choose their positions to maximize the expected trading profits conditional on their information sets $\mathcal{I}_{i}=\left\{\tilde{x}_{i}, \tilde{y}_{i}\right\}$.

The traded asset is a claim on the portion of the aggregate output that remains after 
removing capital providers' share. ${ }^{5}$ Specifically, the aggregate output is

$$
\tilde{Q} \equiv \int_{0}^{1} q\left(k_{j}\right) d j=\tilde{A} \tilde{F} \int_{0}^{1} k_{j} d j=\tilde{A} \tilde{F} K
$$

So, after removing the $\beta$ fraction of $\tilde{Q}$, the remaining $(1-\beta)$ fraction constitutes the cash flow on the risky asset:

$$
\tilde{V} \equiv(1-\beta) \tilde{Q}=(1-\beta) \tilde{A} \tilde{F} K .
$$

A speculator's profit from buying one unit of the asset is given by $\tilde{V}-\tilde{P}$, and similarly, his profit from shorting one unit is $\tilde{P}-\tilde{V}$. So, speculator $i$ chooses demand $d(i)$ to solve:

$$
\max _{d(i) \in[-1,1]} d(i) E\left[(1-\beta) \tilde{A} \tilde{F} K-\tilde{P} \mid \mathcal{I}_{i}\right] .
$$

Since each speculator is atomistic and risk neutral, he will optimally choose to either buy up to the one-unit position limit, or short up to the one-unit position limit. We denote the aggregate demand from speculators as $D \equiv \int_{0}^{1} d(i) d i$, which is the fraction of speculators who buy the asset minus the fraction of those who short the asset.

As in Goldstein, Ozdenoren, and Yuan (2013), to prevent a price that fully reveals the factor $\tilde{f}$ to capital providers, we assume the following noisy supply curve provided by (unmodelled) liquidity traders:

$$
L(\tilde{\xi}, \tilde{P}) \equiv 1-2 \Phi(\tilde{\xi}-\lambda \log \tilde{P}),
$$

where $\tilde{\xi} \sim N\left(0, \tau_{\xi}^{-1}\right)$ (with $\tau_{\xi}>0$ ) is an exogenous demand shock independent of other shocks in the economy. Function $\Phi(\cdot)$ denotes the cumulative standard normal distribution function, which is increasing. Thus, the supply curve $L(\tilde{\xi}, \tilde{P})$ is strictly increasing in the price $\tilde{P}$ and decreasing in the demand shock $\tilde{\xi}$. The parameter $\lambda$ captures the elasticity of the supply curve with respect to the price, and it can be interpreted as the liquidity of the market in the sense of price impact: When $\lambda$ is high, the supply is very elastic with respect to the price and thus, the demand from informed speculators can be easily absorbed by noise trading without moving the price very much.

The market clears by equating the aggregate demand $D \equiv \int_{0}^{1} d(i) d i$ from speculators

\footnotetext{
${ }^{5}$ As explained in Goldstein, Ozdenoren, and Yuan (2013), for technical reasons, we do not assume that the asset is a claim on the net return from the investment.
} 
with the noisy supply $L(\tilde{\xi}, \tilde{P})$ :

$$
D=L(\tilde{\xi}, \tilde{P}) .
$$

This market clearing condition will determine the equilibrium price $\tilde{P}$.

After completing the description of our model, we can see that our setup differs from the one described by Goldstein, Ozdenoren, and Yuan (2013) in two important ways. First, the investment productivity has two sources of uncertainty $(\tilde{a}$ and $\tilde{f})$ in our model, while it has only one dimensional uncertainty $(\tilde{f})$ in Goldstein, Ozdenoren, and Yuan's (2013) economy. As we mentioned earlier, this two-factor production technology allows us to parsimoniously capture the "mis(match)" channel. Second, the information structure is quite different. Goldstein, Ozdenoren, and Yuan (2013) specify that the noise in speculators' private information contains both an idiosyncratic term and a common term, and by doing so, they can study how traders coordinate on trading on rumors represented by the common error term. In contrast, we have shut down the common noise term in speculators' information to remove speculators' coordination motives. Instead, our analysis has emphasized how capital providers coordinate on real investments through observing the common endogenous price information and the externality in their cost function, which forms the basis of our "externality channel."

\subsection{Equilibrium Definition}

An equilibrium involves the optimal decisions of each player (capital providers and speculators) and the statistical behavior of aggregate variables $(K, D$, and $\tilde{P})$. Each player's optimal decision will be a function of their information sets. For capital providers, their optimal investments $k_{j}^{*}$ will be a function of their information set $\mathcal{I}_{j}=\left\{\tilde{a}, \tilde{s}_{j}, \tilde{P}\right\}$; that is, $k_{j}^{*}=k\left(\tilde{a}, \tilde{s}_{j}, \tilde{P}\right)$. We also expect that the noise terms $\tilde{\varepsilon}_{s, j}$ in the signal $\tilde{s}_{j}$ will wash out after aggregation, and thus the aggregate investment $K$ will be a function of $(\tilde{a}, \tilde{f}, \tilde{P})$ :

$$
K=K(\tilde{a}, \tilde{f}, \tilde{P})=\int_{0}^{1} k\left(\tilde{a}, \tilde{s}_{j}, \tilde{P}\right) d j=E\left[k\left(\tilde{a}, \tilde{s}_{j}, \tilde{P}\right) \mid \tilde{a}, \tilde{f}, \tilde{P}\right]
$$

where the expectation is taken over the noise term $\tilde{\varepsilon}_{s, j}$ conditional on $\{\tilde{a}, \tilde{f}, \tilde{P}\}$.

Similarly, speculators' optimal trading strategies $d_{i}^{*}$ will be a function of their information 
set $\mathcal{I}_{i}=\left\{\tilde{x}_{i}, \tilde{y}_{i}\right\}$; that is, $d_{i}^{*}=d\left(\tilde{x}_{i}, \tilde{y}_{i}\right)$. The aggregate demand $D$ for the risky asset is a function of $\tilde{a}$ and $\tilde{f}$ :

$$
D=D(\tilde{a}, \tilde{f})=\int_{0}^{1} d\left(\tilde{x}_{i}, \tilde{y}_{i}\right) d i=E\left[d\left(\tilde{x}_{i}, \tilde{y}_{i}\right) \mid \tilde{a}, \tilde{f}\right]
$$

where the expectation is taken over the noise terms $\tilde{\varepsilon}_{x, i}$ and $\tilde{\varepsilon}_{y, i}$ conditional on $\{\tilde{a}, \tilde{f}, \tilde{P}\}$.

The market clearing condition (11) will therefore determine the price $\tilde{P}$ as a function of productivity factors $\{\tilde{a}, \tilde{f}\}$ and the noise trading shock $\tilde{\xi}: \tilde{P}=P(\tilde{a}, \tilde{f}, \tilde{\xi})$. An equilibrium is defined formally as follows.

Definition 1 An equilibrium consists of a price function, $P(\tilde{a}, \tilde{f}, \tilde{\xi}): \mathbb{R}^{3} \rightarrow \mathbb{R}$, an investment policy for capital providers, $k\left(\tilde{a}, \tilde{s}_{j}, \tilde{P}\right): \mathbb{R}^{3} \rightarrow \mathbb{R}$, a trading strategy of speculators, $d\left(\tilde{x}_{i}, \tilde{y}_{i}\right): \mathbb{R}^{2} \rightarrow[-1,1]$, and the corresponding aggregate investment function $K(\tilde{a}, \tilde{f}, \tilde{P})$, and aggregate demand function for the asset $D(\tilde{a}, \tilde{f})$, such that:

(a) For capital provider $j, k\left(\tilde{a}, \tilde{s}_{j}, \tilde{P}\right)$ solves (4);

(b) For speculator $i, d\left(\tilde{x}_{i}, \tilde{y}_{i}\right)$ solves (9);

(c) The market clearing condition (11) is satisfied; and

(d) The aggregate investment and demand are given by (12) and (13), respectively.

\section{Equilibrium Characterization}

In this section, we construct an equilibrium, and it turns out that solving this equilibrium boils down to a fixed-point problem of characterizing the weight $\phi$ that speculators put on the signal $\tilde{y}_{i}$ about factor $\tilde{f}$ when they trade the risky asset. Specifically, we first conjecture a trading strategy of speculators and use the market clearing condition to determine the asset price and hence the information that capital providers can learn from the price. We then update capital providers' beliefs and characterize their investment rule, which in turn determines the cash flow of the traded asset. Finally, given the implied price and cash flow in the first two steps, we solve for speculators' optimal trading strategy, which compares with the initial conjectured trading strategy to complete the fixed-point loop. 


\subsection{The Information that Capital Providers Learn from the Price}

We conjecture that speculators buy the asset if and only if a linear combination of their signals is above a cutoff $g$, and sell it otherwise. That is, speculators buy the asset whenever $\tilde{x}_{i}+\phi \tilde{y}_{i}>g$, where $\phi$ and $g$ are two endogenous parameters that will be determined in equilibrium. Note that $\tilde{x}_{i}+\phi \tilde{y}_{i}>g$ is equivalent to $\frac{\tilde{\varepsilon}_{x, i}+\phi \tilde{\varepsilon}_{y, i}}{\sqrt{\tau_{x}^{-1}+\phi^{2} \tau_{y}^{-1}}}>\frac{g-(\tilde{a}+\phi \tilde{f})}{\sqrt{\tau_{x}^{-1}+\phi^{2} \tau_{y}^{-1}}}$, and hence speculators' aggregate purchase can be characterized by $1-\Phi\left(\frac{g-(\tilde{a}+\phi \tilde{f})}{\sqrt{\tau_{x}^{-1}+\phi^{2} \tau_{y}^{-1}}}\right)$. Similarly, their aggregate selling is $\Phi\left(\frac{g-(\tilde{a}+\phi \tilde{f})}{\sqrt{\tau_{x}^{-1}+\phi^{2} \tau_{y}^{-1}}}\right)$. Thus, the net holding from speculators is:

$$
D(\tilde{a}, \tilde{f})=1-2 \Phi\left(\frac{g-(\tilde{a}+\phi \tilde{f})}{\sqrt{\tau_{x}^{-1}+\phi^{2} \tau_{y}^{-1}}}\right) .
$$

The market clearing condition (11) together with equations (10) and (14) indicate that

$$
1-2 \Phi\left(\frac{g-(\tilde{a}+\phi \tilde{f})}{\sqrt{\tau_{x}^{-1}+\phi^{2} \tau_{y}^{-1}}}\right)=1-2 \Phi(\tilde{\xi}-\lambda \log \tilde{P}),
$$

which implies that the equilibrium price is given by:

$$
\tilde{P}=\exp \left(\frac{1}{\lambda \sqrt{\tau_{x}^{-1}+\phi^{2} \tau_{y}^{-1}}} \tilde{a}+\frac{\phi}{\lambda \sqrt{\tau_{x}^{-1}+\phi^{2} \tau_{y}^{-1}}} \tilde{f}+\frac{\tilde{\xi}}{\lambda}-\frac{g}{\lambda \sqrt{\tau_{x}^{-1}+\phi^{2} \tau_{y}^{-1}}}\right) .
$$

Recall that capital provider $j$ has the information set $\left\{\tilde{a}, \tilde{s}_{j}, \tilde{P}\right\}$. So, given the realization of $\tilde{a}$, the price $\tilde{P}$ is equivalent to the following signal in predicting the productivity factor $\tilde{f}$ :

$$
\tilde{s}_{p} \equiv \frac{\lambda \sqrt{\tau_{x}^{-1}+\phi^{2} \tau_{y}^{-1}} \log \tilde{P}-\tilde{a}+g}{\phi}=\tilde{f}+\tilde{\varepsilon}_{p},
$$

where

$$
\tilde{\varepsilon}_{p} \equiv \frac{\sqrt{\tau_{x}^{-1}+\phi^{2} \tau_{y}^{-1}}}{\phi} \tilde{\xi}
$$

which has a precision of

$$
\tau_{p} \equiv \frac{1}{\operatorname{Var}\left(\tilde{\varepsilon}_{p}\right)}=\frac{\phi^{2} \tau_{x} \tau_{y} \tau_{\xi}}{\tau_{y}+\phi^{2} \tau_{x}} .
$$

The endogenous precision $\tau_{p}$ captures how much information capital providers can learn from the price. It is crucially related to real efficiency through guiding capital providers' investment decisions. In (18), we see that $\tau_{p}$ is positively determined by four parameters, $\tau_{\xi}, \tau_{y}, \tau_{x}$, and $\phi$. First, when $\tau_{\xi}$ is high, the noise demand $\tilde{\xi}$ in the market is small, and so the 
price aggregates speculators' private information effectively, providing accurate information to capital providers. Second, when $\tau_{y}$ is high, speculators have precise information about

factor $\tilde{f}$, which makes the price very informative about $\tilde{f}$, all other things being equal. Third, for a similar reason, when speculators trade aggressively on their information about $\tilde{f}$ (i.e., when $\phi$ is high), the price is informative about $\tilde{f}$. Fourth, when $\tau_{x}$ is high, speculators' information $\tilde{x}_{i}$ is close to the true realization of the factor $\tilde{a}$, which is known to capital providers; and thus, capital providers can easily interpret the order flows of speculators and extract information about $\tilde{f}$ from the price.

\subsection{The Optimal Investment Policy of Capital Providers}

The solution to capital provider $j$ 's maximization problem (4) is:

$$
k_{j}^{*}=\frac{\beta \tilde{A} E\left(\tilde{F} \mid \tilde{a}, \tilde{s}_{j}, \tilde{s}_{p}\right)}{c_{0} E\left(K^{\gamma} \mid \tilde{a}, \tilde{s}_{j}, \tilde{s}_{p}\right)},
$$

where we have used the fact that capital provider $j$ 's information set is $\mathcal{I}_{j}=\left\{\tilde{a}, \tilde{s}_{j}, \tilde{P}\right\}=$ $\left\{\tilde{a}, \tilde{s}_{j}, \tilde{s}_{p}\right\}$.

We conjecture the investment rule takes the following form:

$$
k_{j}^{*}=\exp \left(h_{0}+h_{a} \tilde{a}+h_{s} \tilde{s}_{j}+h_{p} \tilde{s}_{p}\right)
$$

where the coefficients $h$ 's will be endogenously determined. By equation (20), we can compute:

$$
K=\int_{0}^{1} k_{j}^{*} d j=\exp \left[\left(h_{0}+\frac{h_{s}^{2}}{2 \tau_{s}}\right)+h_{a} \tilde{a}+h_{s} \tilde{f}+h_{p} \tilde{s}_{p}\right] .
$$

Then, using the expressions of $K, \tilde{s}_{j}$ and $\tilde{s}_{p}$ in (21), (5) and (16), we can can compute $E\left(K^{\gamma} \mid \tilde{a}, \tilde{s}_{j}, \tilde{s}_{p}\right)$ and $E\left(\tilde{F} \mid \tilde{a}, \tilde{s}_{j}, \tilde{s}_{p}\right)$, which are in turn plugged into (19), yielding:

$$
k_{j}^{*}=\exp \left\{\begin{array}{c}
{\left[\log \frac{\beta}{c_{0}}+\frac{1}{2} \frac{1-\left(\gamma h_{s}\right)^{2}}{\tau_{f}+\tau_{s}+\tau_{p}}-\gamma\left(h_{0}+\frac{h_{s}^{2}}{2 \tau_{s}}\right)\right]} \\
+\left(1-\gamma h_{a}\right) \tilde{a}+\frac{\left(1-\gamma h_{s}\right) \tau_{s}}{\tau_{f}+\tau_{s}+\tau_{p}} \tilde{s}_{j}+\left[\frac{\left(1-\gamma h_{s}\right) \tau_{p}}{\tau_{f}+\tau_{s}+\tau_{p}}-\gamma h_{p}\right] \tilde{s}_{p}
\end{array}\right\} .
$$

Comparing (22) with the conjectured investment rule in (20), we can form a system of four equations in terms of four unknowns $h_{0}, h_{a}, h_{s}$ and $h_{p}$. Solving this system, we can compute the coefficients $h$ 's in terms of the endogenous $\tau_{p}$ (and hence $\phi$ through (18)) and 
other exogenous parameters as follows:

$$
\begin{aligned}
& h_{0}=\frac{1}{1+\gamma}\left[\log \frac{\beta}{c_{0}}+\frac{1}{2\left(\tau_{f}+\tau_{p}+\tau_{s}+\gamma \tau_{s}\right)}\right] \\
& h_{a}=\frac{1}{1+\gamma}, \\
& h_{s}=\frac{\tau_{s}}{\tau_{f}+\tau_{p}+\tau_{s}+\gamma \tau_{s}}, \\
& h_{p}=\frac{\tau_{p}}{(1+\gamma)\left(\tau_{f}+\tau_{p}+\tau_{s}+\gamma \tau_{s}\right)} .
\end{aligned}
$$

In (23)-(26), we find that the externality parameter $\gamma$ in capital providers' cost function causes them to invest less aggressively (i.e., the coefficients $h$ 's become smaller). Take the loading $h_{s}$ on private signal $\tilde{s}_{j}$ in $(25)$ as an example. In the absence of externality (i.e., $\gamma=0$ ), speculators' investment rule is proportional to $E\left(\tilde{F} \mid \tilde{a}, \tilde{s}_{j}, \tilde{s}_{p}\right)$ in (19). In capital provider $j$ 's information set $\left\{\tilde{a}, \tilde{s}_{j}, \tilde{s}_{p}\right\}$, the two signals $\left\{\tilde{s}_{j}, \tilde{s}_{p}\right\}$ are useful in predicting $\tilde{f}$ - i.e., $E\left(\tilde{f} \mid \tilde{a}, \tilde{s}_{j}, \tilde{s}_{p}\right)=\frac{\tau_{s}}{\tau_{f}+\tau_{p}+\tau_{s}} \tilde{s}_{j}+\frac{\tau_{p}}{\tau_{f}+\tau_{p}+\tau_{s}} \tilde{s}_{p}$. So, the loading $h_{s}$ on $\tilde{s}_{j}$ is simply the Bayesian weight $\frac{\tau_{s}}{\tau_{f}+\tau_{p}+\tau_{s}}$. When there is externality in investment (i.e., when $\gamma>0$ ), the loading $h_{s}$ drops to $\frac{\tau_{s}}{\tau_{f}+\tau_{p}+\tau_{s}+\gamma \tau_{s}}$. This is because facing a stronger signal $\tilde{s}_{j}$, capital provider $j$ now knows that the other capital providers will also invest more, which will drive up the investment cost, and as a response, capital provider $j$ will adjust downward his own investment. Also note that $\gamma$ affects $h_{p}$ in (26) more than $h_{s}$ in (25): $\tilde{s}_{p}$ is a common signal to all capital providers, while $\tilde{s}_{j}$ is a private signal; so capital providers are easier to coordinate on $\tilde{s}_{p}$ than $\tilde{s}_{j}$ when adjusting the externality effect on their investment.

\subsection{The Optimal Trading Strategy of Speculators}

Using the expression of $\tilde{P}$ in (15), we can compute the expected price conditional on speculator $i$ 's information set $\left\{\tilde{x}_{i}, \tilde{y}_{i}\right\}$ as:

$$
E\left(\tilde{P} \mid \tilde{x}_{i}, \tilde{y}_{i}\right)=\exp \left(b_{0}^{p}+b_{x}^{p} \tilde{x}_{i}+b_{y}^{p} \tilde{y}_{i}\right)
$$


where

$$
\begin{aligned}
b_{0}^{p} & =-\frac{g}{\lambda \sqrt{\tau_{x}^{-1}+\phi^{2} \tau_{y}^{-1}}}+\frac{1}{2 \lambda^{2}}\left(\frac{1}{\tau_{\xi}}+\frac{\frac{1}{\tau_{a}+\tau_{x}}}{\tau_{x}^{-1}+\phi^{2} \tau_{y}^{-1}}+\frac{\phi^{2} \frac{1}{\tau_{f}+\tau_{y}}}{\tau_{x}^{-1}+\phi^{2} \tau_{y}^{-1}}\right) \\
b_{x}^{p} & =\frac{1}{\lambda \sqrt{\tau_{x}^{-1}+\phi^{2} \tau_{y}^{-1}}} \frac{\tau_{x}}{\tau_{a}+\tau_{x}}, \\
b_{y}^{p} & =\frac{\phi}{\lambda \sqrt{\tau_{x}^{-1}+\phi^{2} \tau_{y}^{-1}}} \frac{\tau_{y}}{\tau_{f}+\tau_{y}} .
\end{aligned}
$$

Using (8), (16) and (21), we can compute the expected cash flow on the asset conditional on $\left\{\tilde{x}_{i}, \tilde{y}_{i}\right\}$ as follows:

$$
E\left(\tilde{V} \mid \tilde{x}_{i}, \tilde{y}_{i}\right)=(1-\beta) \exp \left(b_{0}^{v}+b_{x}^{v} \tilde{x}_{i}+b_{y}^{v} \tilde{y}_{i}\right)
$$

where

$$
\begin{aligned}
b_{0}^{v} & =h_{0}+\frac{1}{2}\left[\frac{h_{s}^{2}}{\tau_{s}}+\frac{\left(h_{a}+1\right)^{2}}{\tau_{a}+\tau_{x}}+\frac{\left(h_{s}+h_{p}+1\right)^{2}}{\tau_{f}+\tau_{y}}+\frac{h_{p}^{2}}{\tau_{p}}\right] \\
b_{x}^{v} & =\left(h_{a}+1\right) \frac{\tau_{x}}{\tau_{a}+\tau_{x}}, \\
b_{y}^{v} & =\left(h_{s}+h_{p}+1\right) \frac{\tau_{y}}{\tau_{f}+\tau_{y}} .
\end{aligned}
$$

Speculator $i$ will choose to buy the asset if and only if $E\left(\tilde{V} \mid \tilde{x}_{i}, \tilde{y}_{i}\right)>E\left(\tilde{P} \mid \tilde{x}_{i}, \tilde{y}_{i}\right)$. By (27)-(32), we have:

$$
E\left(\tilde{V} \mid \tilde{x}_{i}, \tilde{y}_{i}\right)>E\left(\tilde{P} \mid \tilde{x}_{i}, \tilde{y}_{i}\right) \Leftrightarrow\left(b_{x}^{v}-b_{x}^{p}\right) \tilde{x}_{i}+\left(b_{y}^{v}-b_{y}^{p}\right) \tilde{y}_{i}>\left(b_{0}^{p}-b_{0}^{v}\right)-\log (1-\beta) .
$$

Recall that we conjecture speculators' trading strategy as buying the asset whenever $\tilde{x}_{i}+$ $\phi \tilde{y}_{i}>g$. So, to be consistent with our initial conjecture, we need $b_{x}^{v}-b_{x}^{p}>0$ in (33). By the expressions of $b_{x}^{v}$ and $b_{x}^{p}$ in (31) and (28), we find that:

$$
b_{x}^{v}-b_{x}^{p}>0 \Leftrightarrow \frac{\gamma+2}{\gamma+1}>\frac{1}{\lambda \sqrt{\tau_{x}^{-1}+\phi^{2} \tau_{y}^{-1}}} .
$$

Apparently, if

$$
\lambda \geq \sqrt{\tau_{x}}
$$

then $\frac{1}{\lambda \sqrt{\tau_{x}^{-1}+\phi^{2} \tau_{y}^{-1}}} \leq 1$ and so $b_{x}^{v}-b_{x}^{p}>0$ is satisfied.

Hence, given $b_{x}^{v}-b_{x}^{p}>0$, we can rewrite (33) as $\tilde{x}_{i}+\frac{b_{y}^{v}-b_{y}^{p}}{b_{x}^{v}-b_{x}^{p}} \tilde{y}_{i}>\frac{\left(b_{0}^{p}-b_{0}^{v}\right)-\log (1-\beta)}{b_{x}^{v}-b_{x}^{p}}$. Comparing 
this with the initial conjectured trading rule, we have that in equilibrium,

$$
\begin{aligned}
\phi & =\frac{b_{y}^{v}-b_{y}^{p}}{b_{x}^{v}-b_{x}^{p}} \\
g & =\frac{\left(b_{0}^{p}-b_{0}^{v}\right)-\log (1-\beta)}{b_{x}^{v}-b_{x}^{p}} .
\end{aligned}
$$

Plugging the expressions of $b$ 's, $h$ 's and $\tau_{p}$ into (34), we have one equation determining the equilibrium weight $\phi$ that speculators put on the information about $\tilde{f}$. Analyzing this equation, we find that there always exists a positive solution by the intermediate value theorem. This in turn delivers the following existence proposition.

Proposition 1 When the noisy supply is sufficiently elastic (i.e., when $\lambda \geq \sqrt{\tau_{x}}$ ), there exists an equilibrium characterized by the weight $\phi>0$ that speculators put on the private signals $\tilde{y}_{i}$ about productivity factor $\tilde{f}$.

\section{Market Efficiency and Real Efficiency}

In this section, we first define the concepts of market efficiency and real efficiency and then explain how these two efficiency concepts are connected in general.

\subsection{Market Efficiency}

Market efficiency concerns the extent to which asset prices are informative about the value of traded assets. For example, Fama (1970, p. 383) writes: "A market in which prices always 'fully reflect' available information is called 'efficient."' As we mentioned in the Introduction, it is sometimes labeled as "informational efficiency" or "price efficiency" in the literature. We use these terms interchangeably.

In our model, the cash flow $\tilde{V}$ and the price $\tilde{P}$ of the traded asset are given by equations (8) and (15), respectively. Both variables follow a lognormal distribution. To maintain linearity, we take $\operatorname{logs}$ of $\tilde{V}$ and $\tilde{P}$ - i.e., $\tilde{v} \equiv \log \tilde{V}$ and $\tilde{p} \equiv \log \tilde{P}$ - and define market efficiency as the correlation coefficient between $\tilde{v}$ and $\tilde{p}$ :

$$
M E \equiv \frac{\operatorname{Cov}(\tilde{v}, \tilde{p})}{\sqrt{\operatorname{Var}(\tilde{v}) \operatorname{Var}(\tilde{p})}} .
$$


This is consistent with Grossman and Stiglitz (1980, p. 399) who suggest using squared correlation coefficient between the price and the fundamental to measure the informativeness of the price system.

The literature has also employed other market efficiency measures. For example, another oft-adopted measure is the precision of asset payoff conditional on its price - i.e., $\frac{1}{\operatorname{Var}(\tilde{v} \mid \tilde{p})}-$ in models with exogenous asset cash flows (e.g., Peress, 2010; Ozsoylev and Walden, 2011). This alternative measure is in line with our measure. To see this, note that the cash flow in our model is endogenous, so it is natural to normalize the alternative measure $\frac{1}{\operatorname{Var}(\tilde{v} \mid \tilde{p})}$ by the variance $\operatorname{Var}(\tilde{v})$ of the endogenous cash flow; that is, $\frac{\operatorname{Var}(\tilde{v})}{\operatorname{Var}(\tilde{v} \mid \tilde{p})}$. By the property of normal distributions, we can show that $\frac{\operatorname{Var}(\tilde{v})}{\operatorname{Var}(\tilde{v} \mid \tilde{p})}$ is simply a monotonic transformation of $M E$ :

$$
\frac{\operatorname{Var}(\tilde{v})}{\operatorname{Var}(\tilde{v} \mid \tilde{p})}=\frac{\operatorname{Var}(\tilde{v})}{\operatorname{Var}(\tilde{v})-\frac{(\operatorname{Cov}(\tilde{v}, \tilde{p}))^{2}}{\operatorname{Var}(\tilde{p})}}=\frac{1}{1-\frac{(\operatorname{Cov}(\tilde{v}, \tilde{p}))^{2}}{\operatorname{Var}(\tilde{v}) \operatorname{Var}(\tilde{p})}}=\frac{1}{1-M E^{2}} .
$$

Another advantage of using the correlation coefficient between $\tilde{v}$ and $\tilde{p}$ to measure market efficiency is that it has a normalization flavor since it is always bounded between 0 and 1 . In the Appendix, we compute a more explicit expression of $M E$.

\subsection{Real Efficiency}

We follow Goldstein, Ozdenoren, and Yuan (2013) and measure real efficiency by the ex-ante expected net benefit of investment evaluated in equilibrium. That is, real efficiency is defined as:

$$
R E \equiv E(\tilde{Q}-\tilde{C})
$$

where $\tilde{Q}=\tilde{A} \tilde{F} K$ is the aggregate output defined by $(7), \tilde{C} \equiv \int c\left(k_{j} ; K\right) d j$ is the aggregate cost, and the expectation operator is taken over $(\tilde{a}, \tilde{f}, \tilde{P})$ with respect to their ex-ante distributions.

Direct computation shows

$$
\begin{aligned}
& E(\tilde{Q})=\exp \left[h_{0}+\frac{\left(h_{a}+1\right)^{2}}{2 \tau_{a}}+\frac{\left(h_{s}+h_{p}+1\right)^{2}}{2 \tau_{f}}+\frac{h_{p}^{2}}{2 \tau_{p}}+\frac{h_{s}^{2}}{2 \tau_{s}}\right], \\
& E(\tilde{C})=\frac{c_{0}}{2} \exp \left[\begin{array}{c}
(\gamma+2) h_{0}+\frac{(\gamma+2)^{2} h_{a}^{2}}{2 \tau_{a}}+\frac{(\gamma+2)^{2}\left(h_{s}+h_{p}\right)^{2}}{2 \tau_{f}} \\
+\frac{(\gamma+2)^{2} h_{p}^{2}}{2 \tau_{p}}+\frac{(\gamma+4) h_{s}^{2}}{2 \tau_{s}}
\end{array}\right] .
\end{aligned}
$$


Inserting the expressions of $h$ 's in (23)-(26) into the above expressions, we can compute real efficiency as follows:

$$
R E=\left(\frac{\beta}{c_{0}}\right)^{\frac{1}{1+\gamma}}\left(1-\frac{\beta}{2}\right) \exp \left[\begin{array}{c}
\frac{1}{2 \tau_{a}}\left(\frac{\gamma+2}{\gamma+1}\right)^{2} \\
+\frac{\frac{\tau_{f}+\tau_{p}}{1+\gamma}+\frac{\tau_{p}}{(1+\gamma)^{2}}+2 \tau_{s}+\left(\tau_{f}+\frac{2+\gamma}{1+\gamma} \tau_{p}+(2+\gamma) \tau_{s}\right)^{2} \frac{1}{\tau_{f}}}{2\left(\tau_{f}+\tau_{p}+\tau_{s}+\gamma \tau_{s}\right)^{2}}
\end{array}\right] .
$$

\subsection{The Link Between the Two Efficiency Concepts}

As we discussed in the Introduction, regulators and academics often see promoting market efficiency as one important goal, because market efficiency is generally believed to be a good proxy for real efficiency. The idea can be best illustrated by Figure 1. We care about how trading in financial markets can affect market efficiency and real efficiency. In our model, there are four parameters related to trading: two demand parameters $\tau_{x}$ and $\tau_{y}$ (the precision of speculators' private information), and two supply parameters $\tau_{\xi}$ and $\lambda$ (the level and elasticity of noise supply). Exploring the efficiency implications of changing trading environment can be implemented by conducting comparative statics with respect to these primitive trading parameters. For example, an increase in the information precision $\tau_{x}$ and $\tau_{y}$ can be interpreted as traders acquiring more information at a lower informationacquisition cost (e.g., due to more disclosure, more stringent accounting/auditing rules, or to advanced information-production technology).

\section{[INSERT FIGURE 1 HERE.]}

Suppose for some exogenous reasons, a trading parameter changes, which improves market efficiency (i.e., arrow (i) in Figure 1). We care about whether real efficiency also improves, so that market efficiency is a good proxy for real efficiency (i.e., whether link (iv) holds). In order for this to be true in our economy, we need two more links to hold simultaneously: (1) the change in the financial market improves the accuracy $\tau_{p}$ of information $\tilde{f}$ that capital providers as real decision makers care about (i.e., arrow (ii)); and (2) the increased precision $\tau_{p}$ that each capital provider acquires can be translated into real efficiency for the whole economy (i.e., arrow (iii)). (Of course, if $\tau_{p}$ decreases with the trading parameter, and if $R E$

decreases with $\tau_{p}$, we still have that real efficiency increases.) These two links correspond to the "(mis)match channel" and the "externality channel," respectively. 
In mathematics, note that any trading parameter $m \in\left\{\tau_{x}, \tau_{y}, \tau_{\xi}, \lambda\right\}$ affects real efficiency $R E$ in equation (40) only through its effect on $\tau_{p}$, and thus, by the chain rule, we can express the effect of $m$ on $R E$ as follows:

$$
\frac{\partial R E}{\partial m}=\underbrace{\frac{\partial R E}{\partial \tau_{p}}}_{\text {Externality channel; Proposition } 3} \times \underbrace{\frac{\partial \tau_{p}}{\partial m} .}_{\text {(Mis)Match channel; Propositions 4,5 }}
$$

In the following two sections, we will examine whether the externality channel and the (mis)match channel hold, respectively. In Section 5, we will examine whether and when increasing $\tau_{p}$ will increase real efficiency $R E$ (the externality channel). In Section 6, we will take $\tau_{x}$ and $\tau_{y}$ as an example, and examine whether and when they affect $\tau_{p}$ and $M E$ in the same direction (the (mis)match channel).

\section{Externality and Real Efficiency: The Externality Chan- nel}

\subsection{First-Best Investment Policy}

We first consider a benchmark economy in which a social planner fully internalizes externality, and show that in this economy increasing the information precision $\tau_{p}$ in the price will always improve real efficiency. Specifically, we keep the information structure as before, and in particular, in the interim stage we allow capital providers to access to only their private signals and the price. We still restrict our analysis to loglinear investment rules specified by (20), $k_{j}=\exp \left(h_{0}+h_{a} \tilde{a}+h_{s} \tilde{s}_{j}+h_{p} \tilde{s}_{p}\right)$. Then, we assume that a social planner chooses the coefficients $h$ 's to maximize the real efficiency measure $R E$ in (37), which depends on $h$ 's

through the characterizations of $E(\tilde{Q})$ and $E(\tilde{C})$ in $(38)$ and (39), respectively. We use the superscript "opt" to denote the resulting optimal levels of variables of interest, and the results are formally characterized in the following proposition.

Proposition 2 (a) The investment policy that maximizes real efficiency is

$$
k_{j}^{o p t}=\exp \left(h_{0}^{o p t}+h_{a}^{o p t} \tilde{a}+h_{s}^{o p t} \tilde{s}_{j}+h_{p}^{o p t} \tilde{s}_{p}\right),
$$


where

$$
\begin{aligned}
h_{0}^{o p t} & =\frac{1}{1+\gamma}\left[-\frac{1}{2} \frac{4 \tau_{f}-4 \tau_{p}-4 \tau_{s}+3 \gamma^{2} \tau_{s}+\gamma^{3} \tau_{s}}{\left(2 \tau_{f}+2 \tau_{p}+2 \tau_{s}+3 \gamma \tau_{s}+\gamma^{2} \tau_{s}\right)^{2}}-\left(\log (\gamma+2)+\log \frac{c_{0}}{2}\right)\right], \\
h_{a}^{o p t} & =\frac{1}{1+\gamma}, \\
h_{s}^{o p t} & =\frac{(\gamma+2) \tau_{s}}{2\left(\tau_{f}+\tau_{p}+\tau_{s}\right)+\gamma \tau_{s}(\gamma+3)}, \\
h_{p}^{o p t} & =\frac{\tau_{p}}{(1+\gamma)\left(\tau_{f}+\tau_{p}+\tau_{s}+\gamma \tau_{s} \frac{\gamma+3}{2}\right)} .
\end{aligned}
$$

(b) The resulting real efficiency $R E^{\text {opt }}$ increases with the precision $\tau_{p}$ of the information about $\tilde{f}$ contained in the price.

The result in Part (b) is intuitive. Since the information about $\tilde{f}$ helps capital providers to make real decisions, when the price provides more information about $\tilde{f}$, the social planner can equip capital providers with this better information, thereby improving the overall real efficiency.

\subsection{Inefficient Use of Information and Investment Efficiency}

Now let us examine how increasing $\tau_{p}$ affects real efficiency in our competitive economy. Taking derivative of the expression of $R E$ in (40) with respect to $\tau_{p}$ delivers:

$$
\frac{\partial R E}{\partial \tau_{p}} \propto-\tau_{s} \gamma^{2}+\left(\tau_{f}+\tau_{p}+\tau_{s}\right) \gamma+2\left(\tau_{f}+\tau_{p}+\tau_{s}\right)
$$

This suggests that unlike Part (b) of Proposition 2, in the competitive economy in which capital providers do not internalize externality, increasing the precision $\tau_{p}$ of information (that each capital provider can learn from the price regarding factor $\tilde{f}$ ) can actually reduce real efficiency. This will occur if and only if the externality level $\gamma$ is sufficiently high. This result is formalized in the following proposition.

Proposition 3 When the externality level $\gamma$ in capital providers' cost function is sufficiently low (high), real investment efficiency RE increases (decreases) with the precision $\tau_{p}$ of information that capital providers learn from the price regarding the productivity factor $\tilde{f}$.

In the competitive economy, increasing $\tau_{p}$ has two opposite effects. The first effect is a positive effect. Increasing $\tau_{p}$ simply injects more amount of information into the economy, and as in the first-best benchmark case, each capital provider can learn better the 
underlying productivity factor $\tilde{f}$, which tends to improve real efficiency. In the absence of externality, only this effect is active, and thus increasing $\tau_{p}$ will increase real efficiency when $\gamma$ is sufficiently small.

The second effect is negative. The presence of externality induces "inefficient" use of information in the competitive economy relative to the first-best benchmark, which tends to reduce real efficiency. Specifically, by comparing the coefficients $h_{p}$ and $h_{s}$ on the common price signal $\tilde{s}_{p}$ and on the private signal $\tilde{s}_{j}$ in the investment rule respectively in equations (26) and (25) with those in Proposition 2, we find that in the competitive economy, capital providers overuse the common price information and underuse their private information; that is, $h_{p}>h_{p}^{\text {opt }}$ and $h_{s}<h_{s}^{\text {opt }}$. This is because the common price information helps them to better predict the aggregate investment $K$ which affects their future payoff through the externality in costs - i.e., all capital providers rely on the common price signal to make real investment decisions, and therefore each capital provider can predict this common-signal-based aggregate investment without any error. Since this distortion of using information is driven by externality, a stronger externality makes this distortion more severe (i.e., $\frac{\partial}{\partial \gamma} \frac{h_{p} / h_{s}}{h_{p}^{o p t} / h_{s}^{o p t}}>0$ ).

A higher precision $\tau_{p}$ of the common price signal implies a stronger role of the price signal in determining real efficiency, and thus, when $\gamma$ is sufficiently large so that the inefficient use of information is sufficiently strong, increasing $\tau_{p}$ can harm real efficiency through the channel of inefficient use of information (i.e., the externality channel).

\section{Efficiency Implications of Private Signals: The (Mis)Match Channel}

\subsection{The Effect of $\tau_{x}$}

In this subsection, we conduct comparative statics analysis with respect to parameter $\tau_{x}$, the precision of speculators' information about factor $\tilde{a}$ that capital providers do not care to learn. This corresponds to a thought experiment that speculators can acquire more information about $\tilde{a}$ due to some exogenous shocks to the economy such as changes in disclosure regulation rules or in information-acquisition technology. We focus on economies 
in which $\tau_{x}$ is small, which is arguably empirically relevant, because the whole idea of feedback effects is that traders have imprecise information, but after aggregation, the price can be quite informative.

As $\tau_{x}$ increases, the signal $\tilde{x}_{i}$ becomes more informative about the factor $\tilde{a}$, and thus speculators would like to trade more on this signal than the other signal $\tilde{y}_{i}$ about the other factor $\tilde{f}$. This tends to reduce the relative weight $\phi$ that speculators put on $\tilde{y}_{i}$ in their trading strategies; that is, $\frac{\partial \phi}{\partial \tau_{x}}<0$ when $\tau_{x}$ is small.

The effect of $\tau_{x}$ on the precision $\tau_{p}$ (of information that capital providers can learn from the price) is more subtle. There are two competing forces at work here, as shown by the expression of $\tau_{p}$ in (18). First, there is a positive direct effect: An increase in $\tau_{x}$ makes speculators' information $\tilde{x}_{i}$ closer to the true realization of $\tilde{a}$ which capital providers know and thus can better extract the information about $\tilde{f}$ in the price variations driven by speculators' trading. Second, a change in $\tau_{x}$ also changes the value of $\phi$, which creates an indirect effect on $\tau_{p}$ : Increasing $\tau_{x}$ will reduce $\phi$, and thus speculators trade less aggressively on their information about $\tilde{f}$, reducing the price informativeness about $\tilde{f}$.

We can show that when $\tau_{x}$ is small, the negative indirect effect dominates, so that capital providers learn less about $\tilde{f}$ as $\tau_{x}$ becomes larger. This result can be best understood by examining the case of $\tau_{x}=0$. In this case, the signal $\tilde{x}_{i}$ provides no information regarding $\tilde{a}$, and so they will no longer rely on signal $\tilde{x}_{i}$ in forming trading strategies. As a result, their aggregate trading is only a signal about factor $\tilde{f}$, which makes the price become most informative about $\tilde{f}$ (i.e., $\tau_{p}$ approaches its maximum $\tau_{y} \tau_{\xi}$ ). Thus, at this moment, making $\tau_{x}$ slightly positive will reduce the price informativeness about $\tilde{f}$. That is, $\frac{\partial \tau_{p}}{\partial \tau_{x}}<0$ when $\tau_{x}$ is small.

This implication for $\tau_{p}$ can be directly translated into implication for real efficiency $R E$ by Proposition 3. That is, when the externality level $\gamma$ is small in capital providers' cost functions (so that the externality channel is almost shut down), increasing $\tau_{x}$ will reduce real efficiency through decreasing $\tau_{p}$; and when the externality level $\gamma$ is large in capital providers' cost functions, increasing $\tau_{x}$ will increase real efficiency, since $R E$ negatively changes with $\tau_{p}$.

Regarding the market-efficiency implication of changing $\tau_{x}$, the proof is more compli- 
cated. Still, we can provide a sufficient condition for $M E$ to increase with $\tau_{x}$. That is, when the level of noise trading $\frac{1}{\tau_{\xi}}$ is small in the economy, an increase in $\tau_{x}$ will improve market efficiency. This result is intuitive: Increasing $\tau_{x}$ is equivalent to injecting more information into the economy; when the noise trading level is small, the market aggregates information effectively, and so market efficiency improves. We summarize the above discussions in the following proposition.

Proposition 4 Suppose the supply elasticity $\lambda$ is high. When the precision $\tau_{x}$ of speculators' private signals about factor $\tilde{a}$ is small, an increase in $\tau_{x}$

(a) decreases the relative weight $\phi$ on private signals about the other factor $\tilde{f}$ in speculators' trading strategy (i.e., $\frac{\partial \phi}{\partial \tau_{x}}<0$ );

(b) decreases the precision $\tau_{p}$ that capital providers learn from the price regarding the factor $\tilde{f}$ (i.e., $\left.\frac{\partial \tau_{p}}{\partial \tau_{x}}<0\right)$;

(c) decreases the real investment efficiency $R E$ if there is not much externality in capital providers' cost functions (i.e., $\frac{\partial R E}{\partial \tau_{x}}<0$ if $\gamma$ is small); and

(d) increases the market efficiency $M E$ if the level of noise trading $\frac{1}{\tau_{\xi}}$ is small (i.e., $\frac{\partial M E}{\partial \tau_{x}}>0$ if $\tau_{\xi} \geq 1$ ).

Figure 2 graphically illustrates Proposition 4. Here, we simply set the precision of all random variables (other than $\tilde{\varepsilon}_{x, i}$ of interest) to be 1 ; that is, $\tau_{a}=\tau_{f}=\tau_{y}=\tau_{s}=\tau_{\xi}=1$. We also arbitrarily choose $c_{0}=1$ and $\beta=\frac{1}{2}$. The patterns are quite robust with respect to changes in these parameter values. In this example, we set $\gamma=0$ to remove the externality in capital providers' cost functions, so that the externality channel is shut down and the information precision $\tau_{p}$ that capital providers learn from the price is a good proxy for real efficiency.

\section{[INSERT FIGURE 2 HERE.]}

We see that as Proposition 4 predicts, when $\tau_{x}$ is small, increasing $\tau_{x}$ will decrease $\phi$, $\tau_{p}$ and $R E$, and will increase $M E$. We also find that when $\tau_{x}$ is relatively large, $\tau_{p}$ and $R E$ actually increase with $\tau_{x}$. This reflects the two offsetting forces of $\tau_{x}$ on $\tau_{p}$ as we mentioned earlier. One the one hand, increasing $\tau_{x}$ directly increases $\tau_{p}$, and on the other hand, it indirectly decreases $\tau_{p}$ through reducing $\phi$. When $\tau_{x}$ is very small, we know that 
the negative indirect effect dominates. By contrast, when $\tau_{x}$ is very large, the positive direct effect dominates. Again, this can be best understood by looking at the limiting case of $\tau_{x} \rightarrow \infty:$ As $\tau_{x}$ approaches to $\infty$, the factor $\tilde{a}$ almost becomes a common knowledge, and so the order flow of speculators reflects most information about the factor $\tilde{f}$, which benefits capital providers' real decision making.

\subsection{The Effect of $\tau_{y}$}

Now we examine the trading and efficiency effect of parameter $\tau_{y}$, the precision of speculators' information about factor $\tilde{f}$ that capital providers care to learn. An increase in $\tau_{y}$ makes speculators' signals $\tilde{y}_{i}$ more informative about factor $\tilde{f}$, which causes them to trade more aggressively on $\tilde{y}_{i}$; that is, $\frac{\partial \phi}{\partial \tau_{y}}>0$.

Increasing $\tau_{y}$ has two positive effects on the precision $\tau_{p}$ of the information that capital providers can learn from the price. The first is a direct effect: A higher $\tau_{y}$ means that speculators have more accurate information about factor $\tilde{f}$, which in turn makes the price more informative about $\tilde{f}$. The second positive effect occurs through the effect of $\tau_{y}$ on $\phi$. That is, an increase in $\tau_{y}$ causes an increase in $\phi$, namely speculators trade more aggressively

on their information about $\tilde{f}$ and hence the price is informative about $\tilde{f}$. Both effects tend to increase $\tau_{p}$, and so $\frac{\partial \tau_{p}}{\partial \tau_{y}}>0$.

Again, by Proposition 3, the effect on $\tau_{p}$ can be readily translated into the effect on real efficiency $R E$ : When the externality level $\gamma$ is small in capital providers' cost functions, increasing $\tau_{y}$ will increase real efficiency through increasing $\tau_{p}$; and when the externality level $\gamma$ is large in capital providers' cost functions, increasing $\tau_{y}$ will decrease real efficiency through increasing $\tau_{p}$.

Finally, we can show that for sufficiently small $\tau_{y}$, increasing $\tau_{y}$ improves market efficiency in markets with low levels of noise trading (and hence effective information aggregation). The intuition is again the same as in the standard model: Increasing speculators' information precision helps price discovery through their trading, and thus market efficiency gets improved. We summarize the above results in the following proposition.

Proposition 5 Suppose the supply elasticity $\lambda$ is high. Then, an increase in $\tau_{y}$ 
(a) increases the relative weight $\phi$ on private signals about factor $\tilde{f}$ in speculators' trading strategy (i.e., $\frac{\partial \phi}{\partial \tau_{y}}>0$ );

(b) increases the precision $\tau_{p}$ that capital providers learn from the price regarding factor $\tilde{f}$ (i.e., $\left.\frac{\partial \tau_{p}}{\partial \tau_{y}}>0\right)$;

(c) increases the real investment efficiency $R E$ if there is not much externality in capital providers' cost functions (i.e., $\frac{\partial R E}{\partial \tau_{y}}<0$ if $\gamma$ is small); and

(d) for a sufficiently small $\tau_{y}$, increases the market efficiency $M E$ in markets with low levels of noise trading (i.e., for small $\tau_{y}, \frac{\partial M E}{\partial \tau_{y}}>0$ if $\tau_{\xi}$ is large).

Figure 3 graphically illustrates Proposition 5. As in Figure 2, we set the precision of all random variables other than $\tilde{\varepsilon}_{y, i}$ to be 1 ; that is, $\tau_{a}=\tau_{f}=\tau_{x}=\tau_{s}=\tau_{\xi}=1$. We choose $c_{0}=1, \beta=\frac{1}{2}$, and $\gamma=0$. We see that, consistent with Proposition 5 , all four variables $\phi, \tau_{p}, R E$, and $M E$ increase with $\tau_{y}$.

[INSERT FIGURE 3 HERE.]

\subsection{The Importance of Feedback Effects and Two-Dimensional Uncertainty}

We now show that two key features of our model are essential in driving the contrasting implications of $\tau_{x}$ for market efficiency vs. real efficiency in Proposition 4. The first feature is the feedback effect from prices to investments through capital providers learning from prices. The second one is the two dimensional uncertainty $(\tilde{a}$ and $\tilde{f})$ in investment technology and capital providers knowing more about one factor $\tilde{a}$ than the other factor $\tilde{f}$ relative to speculators.

Specifically, we conduct two exercises. In the first exercise, we set $\tau_{f} \rightarrow \infty$ and keep $\tau_{a}<\infty$. In this case, factor $\tilde{f}$ becomes publicly known in the economy and capital providers no longer learn from prices (which therefore shuts down the feature of feedback effects), but speculators still trade on their private information about factor $\tilde{a}$. Capital providers now know perfectly the two factors and make real investments without reference to the price, and thus real efficiency is not affected by $\tau_{x}$ and $\tau_{y}$, the precision of speculators' private information about $\tilde{a}$ and $\tilde{f}$, respectively. Market efficiency does not change with $\tau_{y}$ either, because $\tilde{f}$ is common knowledge in the trading game and prices have already perfectly 
reflected $\tilde{f}$. We can show that market efficiency still improves with $\tau_{x}$ : Recall that now our model degenerates to the standard setup in which cash flows are not affected by prices; since $\tilde{f}$ is public information, speculators trade only on information about $\tilde{a}$, and thus as in the standard model, if they have more precise information about $\tilde{a}$, the price will be closer to the fundamental.

In the second exercise, we set $\tau_{a} \rightarrow \infty$ and keep $\tau_{f}<\infty$, so that factor $\tilde{a}$ becomes public information (which therefore shuts down the feature of two-dimensional uncertainty), but capital providers still learn information regarding $\tilde{f}$. In this economy, given that $\tilde{a}$ becomes public information, speculators no longer rely on private signals $\tilde{x}_{i}$ to make financial investments, and so their trading and prices are not affected by the precision $\tau_{x}$ of their private signals about $\tilde{a}$. As a result, the price informativeness about fundamental $\tilde{f}$ and the learning process of capital providers are not affected by $\tau_{x}$; that is, market and real efficiency measures are not affected by $\tau_{x}$. Since capital providers still learn from prices, as in Proposition 5, both real efficiency and market efficiency improve with the precision $\tau_{y}$ of speculators' private information about $\tilde{f}$.

Formally, we have the following proposition.

Proposition 6 Suppose the supply elasticity $\lambda$ is high.

(a) If $\tau_{f} \rightarrow \infty$ and $\tau_{a}<\infty$, then (i) real efficiency is not affected by $\tau_{x}$ and market efficiency increases with $\tau_{x}$, and (ii) both real efficiency and market efficiency are not affected by $\tau_{y}$; (b) If $\tau_{a} \rightarrow \infty$ and $\tau_{f}<\infty$, then (i) both real efficiency and market efficiency are not affected by $\tau_{x}$, and (ii) market efficiency increases with $\tau_{y}$, and if the externality $\gamma$ is small enough, real efficiency increases with $\tau_{y}$ as well.

\section{An Extension: Capital Providers Do Not Know $\tilde{a}$ Perfectly}

In the model analyzed in previous sections, we have assumed that capital providers know factor $\tilde{a}$ perfectly and only have noisy signals about factor $\tilde{f}$, so that they only care about the price's informational content about $\tilde{f}$. In this section, we extend our model by assuming 
that capital providers do not know both factors perfectly, and show that all our results in Propositions 3-5 go through as long as capital providers wish to learn one productivity factor more than the other.

Specifically, we now endow each capital provider $j$ with two signals

$$
\tilde{z}_{j}=\tilde{a}+\tilde{\varepsilon}_{z, j} \text { and } \tilde{s}_{j}=\tilde{f}+\tilde{\varepsilon}_{s, j}
$$

where $\tilde{\varepsilon}_{z, j} \sim N\left(0,1 / \tau_{z}\right)$ (with $\tau_{z}>0$ ) and $\tilde{\varepsilon}_{s, j} \sim N\left(0,1 / \tau_{s}\right)$ (with $\tau_{s}>0$ ) and they are independent of all other random variables. We keep intact all the other features of the model. Our baseline model corresponds to the case of $\tau_{z}=\infty$.

We still consider trading strategies that speculators buy the asset if and only $\tilde{x}_{i}+\phi \tilde{y}_{i}>g$, where $\phi$ and $g$ are endogenous parameters determined in equilibrium. So, their aggregate demand $D(\tilde{a}, \tilde{f})$ is still given by equation (14), and the market clearing condition still implies a price function $P(\tilde{a}, \tilde{f}, \tilde{\xi})$ in equation (15). However, because now capital providers do not observe $\tilde{a}$ perfectly, the price is no longer a signal about $\tilde{f}$ given by (16); but instead, it is a signal about both $\tilde{a}$ and $\tilde{f}$ as follows:

$$
\tilde{s}_{p}^{e x t}=\frac{\tilde{a}}{\phi}+\tilde{f}+\tilde{\varepsilon}_{p}
$$

where $\tilde{\varepsilon}_{p}$ is still defined by (17).

Each capital provider $j$ 's optimal investment decision is:

$$
k_{j}^{*}=\arg \max _{k_{j}} E\left(\beta \tilde{A} \tilde{F} k_{j}-\frac{c_{0}}{2} k_{j}^{2} K^{\gamma} \mid \tilde{a}, \tilde{s}_{j}, \tilde{s}_{p}^{e x t}\right)=\frac{\beta E\left(\tilde{A} \tilde{F} \mid \tilde{z}_{j}, \tilde{s}_{j}, \tilde{s}_{p}^{\text {ext }}\right)}{c_{0} E\left(K^{\gamma} \mid \tilde{z}_{j}, \tilde{s}_{j}, \tilde{s}_{p}^{\text {ext }}\right)} .
$$

We will conjecture an investment rule of

$$
k_{j}^{*}=\exp \left(h_{0}+h_{z} \tilde{z}_{j}+h_{s} \tilde{s}_{j}+h_{p} \tilde{s}_{p}^{e x t}\right),
$$

where $h$ 's are endogenous parameters. We can show that the characterization of the equilibrium boils down to one equation in terms of the ratio $\frac{h_{s}}{h_{z}}$. However, the complexity of the inference problem in this economy precludes a full analytical characterization of the equilibrium, and therefore we rely on numerical analysis.

In Figure 4, we examine the implications of $\tau_{x}$, the precision of speculators' private information about factor $\tilde{a}$. We still set $\tau_{a}=\tau_{f}=\tau_{y}=1, \beta=\frac{1}{2}$, and $c_{0}=1$. We set $\tau_{z}=5$ and $\tau_{s}=1$, so that capital providers know more about factor $\tilde{a}$ than factor $\tilde{f}$. In the upper Panels (a1)-(a3), we set $\gamma=0$, and in the lower Panels (b1)-(b3), we set $\gamma=5$. 
So, this exercise is a robustness check for Propositions 3 and 4 . We see that the upper panels are consistent with Figure 2: When $\tau_{x}$ is small, increasing $\tau_{x}$ reduces real efficiency and increases market efficiency; when $\tau_{x}$ is large, increasing $\tau_{x}$ increases both efficiency measures. Also, consistent with Proposition 3, we see that once $\gamma$ becomes large, the real efficiency implication gets reversed in the lower panels.

\section{[INSERT FIGURES 4 AND 5 HERE.]}

In Figure 5, we examine the implication of $\tau_{y}$, the precision of speculators' private infor-

mation about factor $\tilde{f}$. The other parameter values are the same as in Figure 4. Again, the upper panels are consistent with Figure 3 - that is, both real and market efficiency increase with $\tau_{y}$ if $\gamma$ is small. In the lower panels where $\gamma$ is large, the implications for real efficiency gets reversed, manifesting that the externality channel is at work.

\section{Conclusion}

Market efficiency is often argued as a good proxy for real efficiency. In this paper, we examine whether and when this argument is valid. We emphasize two channels that determine the link between these two efficiency measures. First, the investment externality among real decision makers can bias the relative weights that they put on the differential private vs. common price signals relative to the first-best benchmark investment policy. When the price becomes more informationally efficient, the precision of the common price information becomes higher, and the efficiency loss caused by the inefficient use of information becomes larger too. This negative effect can be so strong that real efficiency and market efficiency move in opposite directions. Since this effect originates from the externality in investments, we label it as the "externality channel."

Second, at a more basic level, market efficiency and real efficiency are simply two different concepts. Market efficiency concerns whether the price of a traded security accurately reflects its future value, while real efficiency is about whether real decision makers are taking value-maximization actions. These two concepts are aligned (in the absence of externality) only when the information revealed by the price is also the information that real decision makers care to learn the most. We formalize this idea in a two-factor model where real 
decision makers know more about one factor than the other. When a financial market incorporates a lot of information about the factor that real decision makers have already known, market efficiency improves, but real efficiency deteriorates. Only when the market aggregates information that real decision makers care to learn, do market efficiency and real efficiency move in the same direction. We refer to this second channel as the "(mis)match channel."

Our analysis has important implications for regulations and empirical research. Various regulation policies encourage private information acquisition with an intention of improving market efficiency (see the discussions in Gao and Liang (2013)). According to our analysis, the link between market efficiency and real efficiency is delicate. If regulation policies are not carefully designed, it is plausible that real efficiency deteriorates although market efficiency improves. A vast empirical literature is devoted to examining how changes in trading environments (such as caused by regulation rules) affect informational efficiency of financial markets (e.g., see Beyer, Cohen, Lys, and Walther (2010)). It remains interesting to see how real efficiency and market efficiency are related to each other in these empirical settings. A recent study by Bai, Philippon, and Savov (2013) has made considerable progress toward this direction by documenting that both market efficiency and real efficiency (due to learning from prices) have remained stable in the U.S. over the last several decades. We think that theoretical analysis like the one provided in our paper can be helpful in guiding such empirical analysis and interpreting its results, in that our theory attempts to spell out explicitly what is the link between market efficiency and real efficiency and how it depends on the underlying environment.

Before we conclude, we make two remarks about our analysis. Our analysis has focused on real investment efficiency and does not provide a full welfare analysis. Conducting such an analysis would require endogenous noise trading as in Dow and Rahi (2003). We abstract this issue mainly for technical consideration, because otherwise, the loglinear structure of the model cannot be easily maintained. Our analysis can be viewed as the first step toward a full welfare analysis, similar as what we do in the standard economics textbook: Production efficiency is a necessary condition for welfare maximization.

Second, in our analysis, financial markets affect real economy through the channel that real decision makers learn information in the asset price to guide their decisions. This is 
motivated by the empirical findings that firms indeed learn from prices (e.g., Chen, Goldstein, and Jiang, 2007; Bakke and Whited, 2010). There are other channels at work in reality, such as the contracting(incentive) channel that firm managers' contracts are contingent upon the prices and so their incentives to take real decisions are affected by the price (e.g., Holmstrom and Tirole, 1993; Dow and Gorton, 1997; Edmans, Heinle, and Huang, 2013). It would be interesting to examine how introducing these other channels into our framework affects the link between market efficiency and real efficiency. We leave all these important questions for future research.

\section{Appendix: Proofs}

\section{The Notations of $o(\cdot)$ and $O(\cdot)$}

Our proofs are frequently involved with the order argument for a particular process. We here introduce the following two notations, $o(\cdot)$ and $O(\cdot)$. Specifically, consider two univariate functions $f_{1}(x)$ and $f_{2}(x)$ and the process of $x \rightarrow \bar{x}$, where $\bar{x}$ is some real constant or $\pm \infty$.

If $\lim _{x \rightarrow \bar{x}} \frac{f_{1}(x)}{f_{2}(x)}=0$, then we denote $f_{1}=o\left(f_{2}\right)$, meaning that if $f_{1}$ and $f_{2}$ converge then $f_{1}$ converges at a faster rate than $f_{2}$ does. If $\lim _{x \rightarrow \bar{x}} \frac{f_{1}(x)}{f_{2}(x)}$ is bounded (but different from 0 ), then we denote $f_{1}=O\left(f_{2}\right)$, meaning that if $f_{1}$ and $f_{2}$ converge, then they converge at the same rate.

\section{Compute Market Efficiency ME}

By (8), we have

$$
\tilde{v}=\log \tilde{V}=\log (1-\beta)+\left(h_{0}+\frac{h_{s}^{2}}{2 \tau_{s}}\right)+\left(h_{a}+1\right) \tilde{a}+\left(h_{s}+1\right) \tilde{f}+h_{p} \tilde{s}_{p} .
$$

Define the linear log transformation of $\tilde{P}$ in (15) as follows:

$$
\hat{p} \equiv \lambda \sqrt{\tau_{x}^{-1}+\phi^{2} \tau_{y}^{-1}} \tilde{p}+g=\tilde{a}+\phi \tilde{s}_{p}
$$


Then, we can compute:

$$
\begin{aligned}
\Sigma_{v} & \equiv \operatorname{Var}(\tilde{v})=\left(h_{a}+1\right)^{2} \frac{1}{\tau_{a}}+\left(h_{s}+h_{p}+1\right)^{2} \frac{1}{\tau_{f}}+\frac{h_{p}^{2}}{\tau_{p}}, \\
\Sigma_{p} & \equiv \operatorname{Var}(\hat{p})=\frac{1}{\tau_{a}}+\phi^{2}\left(\frac{1}{\tau_{f}}+\frac{1}{\tau_{p}}\right), \\
\Sigma_{v p} & \equiv \operatorname{Cov}(\tilde{v}, \hat{p})=\left(h_{a}+1\right) \frac{1}{\tau_{a}}+\left(h_{s}+h_{p}+1\right) \phi \frac{1}{\tau_{f}}+\frac{h_{p} \phi}{\tau_{p}} .
\end{aligned}
$$

By the expressions of $h$ 's in equations (24)-(26), we can further compute:

$$
\begin{aligned}
\Sigma_{v} & =\frac{(2+\gamma)^{2}}{\tau_{a}(1+\gamma)^{2}}+\frac{\left((1+\gamma) \tau_{f}+(2+\gamma)\left(\tau_{p}+\tau_{s}+\gamma \tau_{s}\right)\right)^{2}+\tau_{f} \tau_{p}}{\tau_{f}\left((1+\gamma)\left(\tau_{f}+\tau_{p}+\tau_{s}+\gamma \tau_{s}\right)\right)^{2}} \\
\Sigma_{v p} & =\frac{\gamma+2}{\gamma+1}\left(\frac{1}{\tau_{a}}+\frac{\phi}{\tau_{f}}\right) .
\end{aligned}
$$

So, market efficiency is $M E=\frac{\Sigma_{v p}}{\sqrt{\Sigma_{v} \Sigma_{p}}}$, where $\Sigma_{p}, \Sigma_{v}$ and $\Sigma_{v p}$ are given by (A4), (A6), and (A7), respectively.

\section{Proof of Proposition 2}

(a) Taking derivative of (37) with respect to $h_{0}, h_{a}, h_{s}$ and $h_{p}$, respectively, yields:

$$
\begin{aligned}
E\left(\tilde{Q}^{o p t}\right) & =E\left(\tilde{C}^{o p t}\right)(\gamma+2), \\
E\left(\tilde{Q}^{o p t}\right) \frac{\left(h_{a}^{o p t}+1\right)}{\tau_{a}} & =E\left(\tilde{C}^{o p t}\right) \frac{(\gamma+2)^{2} h_{a}^{o p t}}{\tau_{a}}, \\
E\left(\tilde{Q}^{o p t}\right)\left(\frac{h_{s}^{o p t}+h_{p}^{o p t}+1}{\tau_{f}}+\frac{h_{s}^{o p t}}{\tau_{s}}\right) & =E\left(\tilde{C}^{o p t}\right)\left[\begin{array}{c}
\frac{(\gamma+2)^{2}\left(h_{s}^{o s t}+h_{p}^{o p t}\right)}{\tau_{f}} \\
+\frac{(\gamma+4) h_{s}^{o p t}}{\tau_{s}}
\end{array}\right], \\
E\left(\tilde{Q}^{o p t}\right)\left(\frac{h_{s}^{o p t}+h_{p}^{o p t}+1}{\tau_{f}}+\frac{h_{p}^{o p t}}{\tau_{p}}\right) & =E\left(\tilde{C}^{o p t}\right)\left[\begin{array}{c}
\frac{(\gamma+2)^{2}\left(h_{s}^{o t}+h_{p}^{o p t}\right)}{\tau_{f}} \\
+\frac{(\gamma+2)^{2} h_{p}^{o p t}}{\tau_{p}}
\end{array}\right] .
\end{aligned}
$$

Plugging (A8) into (A9), we can compute $h_{a}^{\text {opt }}=\frac{1}{\gamma+1}$. Plugging (A8) into (A10) and (A11), we can cancel $E\left(\tilde{Q}^{o p t}\right)$ and $E\left(\tilde{C}^{o p t}\right)$ to obtain two linear functions and compute $h_{s}^{\text {opt }}$ and $h_{p}^{\text {opt }}$. After we compute $h_{a}^{\text {opt }}, h_{s}^{\text {opt }}$, and $h_{p}^{\text {opt }}$, we can plug them back into (A8) to obtain the expression of $h_{0}^{\text {opt }}$.

(b) By the envelope theorem, we have

$$
\frac{d R E^{o p t}}{d \tau_{p}}=E\left(\tilde{Q}^{\text {opt }}\right) \frac{\left(h_{p}^{o p t}\right)^{2}}{2}\left(-\frac{1}{\tau_{p}^{2}}\right)-E(\tilde{C}) \frac{(\gamma+2)^{2}\left(h_{p}^{o p t}\right)^{2}}{2}\left(-\frac{1}{\tau_{p}^{2}}\right) .
$$


Then, using $E\left(\tilde{Q}^{\text {opt }}\right)=E\left(\tilde{C}^{\text {opt }}\right)(\gamma+2)$ in $(\mathrm{A} 8)$, we have

$$
\frac{d R E^{o p t}}{d \tau_{p}}=\frac{\left(h_{p}^{o p t}\right)^{2}}{2 \tau_{p}^{2}}(\gamma+1)(\gamma+2) E\left(\tilde{C}^{o p t}\right)>0
$$

\section{Proof of Proposition 4}

Suppose $\lambda \rightarrow \infty$. We first establish the properties of $\phi$ and $\tau_{p}$ as $\tau_{x} \rightarrow 0$. By (34), when $\lambda \rightarrow \infty, \phi$ is determined by

$$
\phi=\frac{\left(\frac{\tau_{s}}{\tau_{f}+\tau_{p}+\tau_{s}+\gamma \tau_{s}}+\frac{\tau_{p}}{(1+\gamma)\left(\tau_{f}+\tau_{p}+\tau_{s}+\gamma \tau_{s}\right)}+1\right) \frac{\tau_{y}}{\tau_{f}+\tau_{y}}}{\frac{2+\gamma}{1+\gamma} \frac{\tau_{x}}{\tau_{a}+\tau_{x}}}
$$

By the expression of $\tau_{p}$ in (18), we know that $\tau_{p}$ is bounded. As a result, as $\tau_{x} \rightarrow 0$, we have $\phi$ converges to $\infty$ at the rage of $\frac{1}{\tau_{x}}$; that is,

$$
\phi=O\left(\frac{1}{\tau_{x}}\right) \text {. }
$$

So, by (18),

$$
\tau_{p}=\frac{\phi^{2} \tau_{x} \tau_{y} \tau_{\xi}}{\tau_{y}+\phi^{2} \tau_{x}}=\tau_{y} \tau_{\xi}+o(1) .
$$

Proof of Part (a). Applying the implicit function theorem to (A12), we can show:

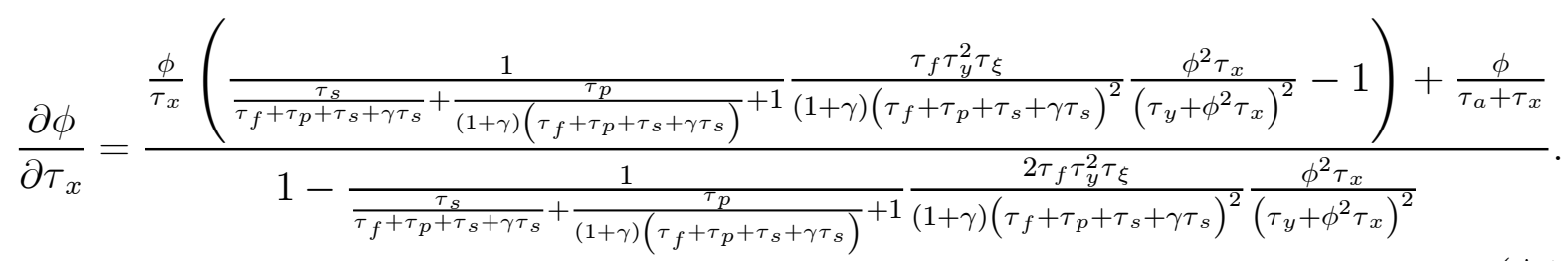

By (A13) and (A14), we have $\lim _{\tau_{x} \rightarrow 0} \phi^{2} \tau_{x}=\infty$ and $\lim _{\tau_{x} \rightarrow 0} \tau_{p}=\tau_{y} \tau_{\xi}$. Inserting these limiting results into the above expression of $\frac{\partial \phi}{\partial \tau_{x}}$ in (A15), we can show

$$
\frac{\partial \phi}{\partial \tau_{x}}=-\frac{\phi}{\tau_{x}}+\frac{\phi}{\tau_{a}}+o\left(\frac{1}{\tau_{x}}\right)
$$

So, as $\tau_{x} \rightarrow 0$, we have $\frac{\partial \phi}{\partial \tau_{x}} \rightarrow-\infty$. 
Proof of Part (b). By the expressions of $\tau_{p}$ in (18) and of $\frac{\partial \phi}{\partial \tau_{x}}$ in (A16), we can compute:

$$
\begin{aligned}
\frac{\partial \tau_{p}}{\partial \tau_{x}} & =\frac{\partial}{\partial\left(\phi^{2} \tau_{x}\right)} \frac{\phi^{2} \tau_{x} \tau_{y} \tau_{\xi}}{\tau_{y}+\phi^{2} \tau_{x}} \frac{\partial\left(\phi^{2} \tau_{x}\right)}{\partial \tau_{x}} \\
& =\tau_{y} \tau_{\xi} \frac{\tau_{y}}{\left(\tau_{y}+\phi^{2} \tau_{x}\right)^{2}}\left(2 \phi \frac{\partial \phi}{\partial \tau_{x}} \tau_{x}+\phi^{2}\right) \\
& =\tau_{y} \tau_{\xi} \frac{\tau_{y}}{\left(\tau_{y}+\phi^{2} \tau_{x}\right)^{2}}\left(2 \phi\left(-\frac{\phi}{\tau_{x}}+\frac{\phi}{\tau_{a}}+o\left(\frac{1}{\tau_{x}}\right)\right) \tau_{x}+\phi^{2}\right) \\
& =\tau_{y} \tau_{\xi} \frac{\tau_{y}}{\left(\tau_{y}+\phi^{2} \tau_{x}\right)^{2}}\left(-\phi^{2}+O(\phi)\right) .
\end{aligned}
$$

Using $\phi=O\left(\frac{1}{\tau_{x}}\right)$ in (A13), we have:

$$
\begin{aligned}
\frac{\partial \tau_{p}}{\partial \tau_{x}} & =\frac{\tau_{y} \tau_{\xi} \tau_{y}}{\left(\tau_{y}+\phi^{2} \tau_{x}\right)^{2}}\left(-\phi^{2}+O(\phi)\right) \\
& =\left(-\frac{\tau_{y} \tau_{y} \tau_{\xi}}{\left(\tau_{y}+\phi^{2} \tau_{x}\right)^{2}}\left(\phi^{2} \tau_{x}\right)^{2}\right) \frac{1}{\left(\phi^{2} \tau_{x}\right) \tau_{x}}+o(1) \\
& =-\frac{\tau_{y} \tau_{y} \tau_{\xi}}{\left(\phi^{2} \tau_{x}\right) \tau_{x}}+o(1)
\end{aligned}
$$

So, as $\tau_{x} \rightarrow 0$, we have $\frac{\partial \tau_{p}}{\partial \tau_{x}}<0$.

Proof of Part (c). This result follows directly from Part (b) and Proposition 3.

Proof of Part (d). By the expression of $\Sigma_{v p}$ in (A7), direct computation shows:

$$
\frac{\partial \log \Sigma_{v p}}{\partial \tau_{x}}=\frac{\frac{\gamma+2}{\tau_{f}(\gamma+1)}}{\left(\frac{2+\gamma}{1+\gamma}\right) \frac{1}{\tau_{a}}+\phi \frac{\gamma+2}{\tau_{f}(\gamma+1)}} \frac{\partial \phi}{\partial \tau_{x}} .
$$

Then using the expression of $\frac{\partial \phi}{\partial \tau_{x}}$ in (A16), we can show:

$$
\frac{\partial \log \Sigma_{v p}}{\partial \tau_{x}}=-\frac{1}{\tau_{x}}+\frac{1}{\tau_{a}}+\frac{\tau_{f}}{\phi \tau_{x} \tau_{a}}+o(1) .
$$

By the expression of $\Sigma_{p}$ in (A4), we have:

$$
\frac{\partial \log \Sigma_{p}}{\partial \tau_{x}}=\frac{2 \phi\left(\frac{1}{\tau_{f}}+\frac{1}{\tau_{p}}\right) \frac{\partial \phi}{\partial \tau_{x}}+\phi^{2}\left(-\frac{1}{\tau_{p}^{2}}\right) \frac{\partial \tau_{p}}{\partial \tau_{x}}}{\frac{1}{\tau_{a}}+\phi^{2}\left(\frac{1}{\tau_{f}}+\frac{1}{\tau_{p}}\right)} .
$$

Then, using equations (A13), (A14), (A16) and (A17), we can compute:

$$
\frac{\partial \log \Sigma_{p}}{\partial \tau_{x}}=2\left(-\frac{1}{\tau_{x}}+\frac{1}{\tau_{a}}\right)+\frac{1}{\tau_{p}} \frac{\tau_{f}}{\tau_{f}+\tau_{p}} \frac{\tau_{y} \tau_{y} \tau_{\xi}}{\left(\phi^{2} \tau_{x}\right) \tau_{x}}+o(1) .
$$


Similarly, by the expression of $\Sigma_{v}$ in (A6),

$$
\frac{\partial \log \Sigma_{v}}{\partial \tau_{x}}=\frac{\frac{3 \tau_{f}+3 \tau_{p}+5 \tau_{s}+2 \gamma \tau_{f}+2 \gamma \tau_{p}+7 \gamma_{s}+2 \gamma^{2} \tau_{s}}{\tau_{f}+\tau_{p}+\tau_{s}+\gamma \tau_{s}}}{\frac{(2+\gamma)^{2}\left(\tau_{f}+\tau_{p}+\tau_{s}+\gamma \tau_{s}\right)^{2}}{\tau_{a}}+\frac{\left((1+\gamma) \tau_{f}+(2+\gamma)\left(\tau_{p}+\tau_{s}+\gamma \tau_{s}\right)\right)^{2}+\tau_{f} \tau_{p}}{\tau_{f}}} \frac{\partial \tau_{p}}{\partial \tau_{x}} .
$$

Then, using $\frac{\partial \tau_{p}}{\partial \tau_{x}}$ in (A17) and the fact that $\tau_{p}$ is bounded, we have:

$$
\frac{\partial \log \Sigma_{v}}{\partial \tau_{x}}=-\frac{\frac{3 \tau_{f}+3 \tau_{p}+5 \tau_{s}+2 \gamma \tau_{f}+2 \gamma \tau_{p}+7 \gamma \tau_{s}+2 \gamma^{2} \tau_{s}}{\tau_{f}+\tau_{p}+\tau_{s}+\gamma \tau_{s}}}{\frac{(2+\gamma)^{2}\left(\tau_{f}+\tau_{p}+\tau_{s}+\gamma \tau_{s}\right)^{2}}{\tau_{a}}+\frac{\left((1+\gamma) \tau_{f}+(2+\gamma)\left(\tau_{p}+\tau_{s}+\gamma \tau_{s}\right)\right)^{2}+\tau_{f} \tau_{p}}{\tau_{f}}} \frac{\tau_{y} \tau_{y} \tau_{\xi}}{\left(\phi^{2} \tau_{x}\right) \tau_{x}}+o(1) .
$$

So, by the definitions of $M E$ in (36), we have:

$$
\frac{\partial \log M E}{\partial \tau_{x}}=\frac{\partial \log \Sigma_{v p}}{\partial \tau_{x}}-\frac{1}{2} \frac{\partial \log \Sigma_{p}}{\partial \tau_{x}}-\frac{1}{2} \frac{\partial \log \Sigma_{v}}{\partial \tau_{x}} .
$$

Plugging equations (A18)-(A20) into the above equation and using the fact of $\tau_{p}=\tau_{y} \tau_{\xi}+$ $o(1)$ in (A14), we have:

$$
\frac{\partial \log M E}{\partial \tau_{x}}=T\left(\phi, \tau_{x}, \tau_{p}, \tau_{f}, \tau_{a}, \tau_{s}, \gamma\right) \frac{\tau_{y} \tau_{p}}{\left(\phi^{2} \tau_{x}\right) \tau_{x}}+o(1) .
$$

where

$$
\begin{aligned}
T\left(\phi, \tau_{x}, \tau_{p}, \tau_{f}, \tau_{a}, \tau_{s}, \gamma\right) \equiv & {\left[\frac{\tau_{f} \phi \tau_{x}}{\tau_{a} \tau_{p} \tau_{y}}-\frac{1}{2 \tau_{p}} \frac{\tau_{f}}{\tau_{f}+\tau_{p}}\right] } \\
& +\frac{1}{2} \frac{\frac{3 \tau_{f}+3 \tau_{p}+5 \tau_{s}+2 \gamma \tau_{f}+2 \gamma \tau_{p}+7 \gamma \tau_{s}+2 \gamma^{2} \tau_{s}}{\tau_{f}+\tau_{p}+\tau_{s}+\gamma \tau_{s}}}{\left[\begin{array}{c}
\frac{(2+\gamma)^{2}\left(\tau_{f}+\tau_{p}+\tau_{s}+\gamma \tau_{s}\right)^{2}}{\tau_{a}} \\
+\frac{\left((1+\gamma) \tau_{f}+(2+\gamma)\left(\tau_{p}+\tau_{s}+\gamma \tau_{s}\right)\right)^{2}+\tau_{f} \tau_{p}}{\tau_{f}}
\end{array}\right]} .
\end{aligned}
$$

So, $\frac{\partial \log M E}{\partial \tau_{x}}>0$ if and only if $T\left(\phi, \tau_{x}, \tau_{p}, \tau_{f}, \tau_{a}, \tau_{s}, \gamma\right)>0$.

By (A12), we have

$$
\phi \tau_{x}=\frac{(1+\gamma) \tau_{f}+(2+\gamma)\left(\tau_{p}+\tau_{s}+\gamma \tau_{s}\right)}{(2+\gamma)\left(\tau_{f}+\tau_{p}+\tau_{s}+\gamma \tau_{s}\right)} \frac{\tau_{y} \tau_{a}}{\tau_{f}+\tau_{y}}+o(1) .
$$

So, the bracketed term in $T\left(\phi, \tau_{x}, \tau_{p}, \tau_{f}, \tau_{a}, \tau_{s}, \gamma\right)$ is:

$$
\begin{aligned}
& \frac{\tau_{f} \phi \tau_{x}}{\tau_{a} \tau_{p} \tau_{y}}-\frac{1}{2 \tau_{p}} \frac{\tau_{f}}{\tau_{f}+\tau_{p}} \\
= & \frac{\tau_{f}}{2 \tau_{p}\left(\tau_{f}+\tau_{y}\right)}\left[\frac{2(1+\gamma)}{2+\gamma}\left(1+\frac{\tau_{s}+\frac{1}{1+\gamma} \tau_{p}}{\tau_{f}+\tau_{p}+(1+\gamma) \tau_{s}}\right)-\frac{\tau_{f}+\tau_{y}}{\tau_{f}+\tau_{p}}\right]+o(1) .
\end{aligned}
$$

So, if

$$
\frac{\tau_{f}+\tau_{y}}{\tau_{f}+\tau_{p}}<1 \Leftrightarrow \tau_{y}<\tau_{p}
$$

then $\frac{\tau_{f} \phi \tau_{x}}{\tau_{a} \tau_{p} \tau_{y}}-\frac{1}{2 \tau_{p}} \frac{\tau_{f}}{\tau_{f}+\tau_{p}}>0$, and hence $T\left(\phi, \tau_{x}, \tau_{p}, \tau_{f}, \tau_{a}, \tau_{s}, \gamma\right)>0$. Given $\tau_{p}=\tau_{y} \tau_{\xi}+o(1)$ 
in (A14), we know

$$
\tau_{y}<\tau_{p} \Leftrightarrow \tau_{\xi}>1 \text {, as } \tau_{x} \rightarrow 0 .
$$

\section{Proof of Proposition 5}

Proof of Parts (a)-(c). By the expression of $\tau_{p}$ in (18), we can show

$$
\frac{\partial \tau_{p}}{\partial \tau_{y}}=\frac{2 \tau_{p} \tau_{y}}{\phi\left(\tau_{y}+\phi^{2} \tau_{x}\right)} \frac{\partial \phi}{\partial \tau_{y}}+\frac{\tau_{p} \phi^{2} \tau_{x}}{\tau_{y}\left(\tau_{y}+\phi^{2} \tau_{x}\right)} .
$$

When $\lambda$ is large, $\phi$ is still determined by (A12). Using the implicit function theorem and the expression of $\frac{\partial \tau_{p}}{\partial \tau_{y}}$ in (A26), we have

$$
\frac{\partial \phi}{\partial \tau_{y}}=\frac{\phi\left(\frac{\tau_{p} \phi^{2} \tau_{x}}{\left((1+\gamma) \tau_{f}+(2+\gamma)\left(\tau_{p}+\tau_{s}+\gamma \tau_{s}\right)\right)\left(\tau_{f}+\tau_{p}+\tau_{s}+\gamma \tau_{s}\right)\left(\tau_{y}+\phi^{2} \tau_{x}\right)}+\frac{1}{\tau_{f}+\tau_{y}}\right) \frac{\tau_{f}}{\tau_{y}}}{1-\frac{2 \tau_{p} \tau_{y} \tau_{f}}{\left(\tau_{y}+\phi^{2} \tau_{x}\right)\left((1+\gamma) \tau_{f}+(2+\gamma)\left(\tau_{p}+\tau_{s}+\gamma \tau_{s}\right)\right)\left(\tau_{f}+\tau_{p}+\tau_{s}+\gamma \tau_{s}\right)}} .
$$

Since $\left(\tau_{y}+\phi^{2} \tau_{x}\right)>\tau_{y},(2+\gamma)\left(\tau_{p}+\tau_{s}+\gamma \tau_{s}\right)>2 \tau_{p}$, and $\left(\tau_{f}+\tau_{p}+\tau_{s}+\gamma \tau_{s}\right)>\tau_{f}$, we know $\left(\tau_{y}+\phi^{2} \tau_{x}\right)\left((1+\gamma) \tau_{f}+(2+\gamma)\left(\tau_{p}+\tau_{s}+\gamma \tau_{s}\right)\right)\left(\tau_{f}+\tau_{p}+\tau_{s}+\gamma \tau_{s}\right)>2 \tau_{p} \tau_{y} \tau_{f}$, and thus the denominator in (A27) is positive. Therefore, $\frac{\partial \phi}{\partial \tau_{y}}>0$ in Part (a). By (A26), we have $\frac{\partial \tau_{p}}{\partial \tau_{y}}>0$ in Part (b) as well. The result on real efficiency in Part (c) follows directly from $\frac{\partial \tau_{p}}{\partial \tau_{y}}>0$ and Proposition 3 .

Proof of Part (d). Using equations (A7), (A4), and (A6), we can compute:

$$
\begin{aligned}
& \frac{\partial \log \Sigma_{v p}}{\partial \tau_{y}}= \frac{1}{\frac{1}{\tau_{a}}+\frac{\phi}{\tau_{f}} \frac{1}{\tau_{f}} \frac{\partial \phi}{\partial \tau_{y}},} \\
& \frac{\partial \log \Sigma_{p}}{\partial \tau_{y}}=\frac{2 \phi\left(\frac{1}{\tau_{f}}+\frac{1}{\tau_{p}}\right) \frac{\partial \phi}{\partial \tau_{y}}+\phi^{2}\left(-\frac{1}{\tau_{p}^{2}}\right) \frac{\partial \tau_{p}}{\partial \tau_{y}}}{\frac{1}{\tau_{a}}+\phi^{2}\left(\frac{1}{\tau_{f}}+\frac{1}{\tau_{p}}\right)}, \\
& \frac{\partial \log \Sigma_{v}}{\partial \tau_{y}}=\frac{\frac{1}{\tau_{f}(1+\gamma)^{2}} \frac{\tau_{f}\left(3 \tau_{f}+3 \tau_{p}+5 \tau_{s}+2 \gamma \tau_{f}+2 \gamma \tau_{p}+7 \tau_{s}+2 \gamma^{2} \tau_{s}\right)}{\left(\tau_{f}+\tau_{p}+\tau_{s}+\gamma \tau_{s}\right)^{3}}}{\frac{(2+\gamma)^{2}}{\tau_{a}(1+\gamma)^{2}}+\frac{\left((1+\gamma) \tau_{f}+(2+\gamma)\left(\tau_{p}+\tau_{s}+\gamma \tau_{s}\right)\right)^{2}+\tau_{f} \tau_{p}}{\tau_{f}\left((1+\gamma)\left(\tau_{f}+\tau_{p}+\tau_{s}+\gamma \tau_{s}\right)\right)^{2}}} \frac{\partial \tau_{p}}{\partial \tau_{y}} .
\end{aligned}
$$

Now let us check their orders as $\tau_{y} \rightarrow 0$. By (A12), we have

$$
\phi=\frac{(\gamma+1)\left(\tau_{a}+\tau_{x}\right)\left(\tau_{f}+2 \tau_{s}+\gamma \tau_{s}\right)}{\tau_{f} \tau_{x}(\gamma+2)\left(\tau_{f}+\tau_{s}+\gamma \tau_{s}\right)} \tau_{y}+o\left(\tau_{y}\right)
$$

and by (18),

$$
\tau_{p}=\phi^{2} \tau_{x} \tau_{\xi}+o\left(\tau_{y}^{2}\right) .
$$


So, the expression of $\frac{\partial \phi}{\partial \tau_{y}}$ in (A27) implies:

$$
\frac{\partial \phi}{\partial \tau_{y}}=\frac{\phi}{\tau_{y}}+o(1)
$$

By (A32), (A33) and (A26), we have:

$$
\frac{\partial \tau_{p}}{\partial \tau_{y}}=\frac{2 \phi^{2} \tau_{x} \tau_{\xi}}{\tau_{y}}+o\left(\tau_{y}\right) .
$$

Equations (A28) and (A33) imply:

$$
\frac{\partial \log \Sigma_{v p}}{\partial \tau_{y}}=\frac{\tau_{a}}{\tau_{f}} \frac{\phi}{\tau_{y}}+o(1) .
$$

Equations (A26), (A29), (A32) and (A33) imply:

$$
\frac{\partial \log \Sigma_{p}}{\partial \tau_{y}}=\frac{\phi^{2} \tau_{a} \tau_{x}}{\tau_{y}^{2}\left(\tau_{a}+\tau_{x} \tau_{\xi}\right)}+o(1) .
$$

Equations (A30), (A32) and (A34) imply:

$$
\frac{\partial \log \Sigma_{v}}{\partial \tau_{y}}=O\left(\tau_{y}\right) .
$$

So, by the expression of $M E$ in (36):

$$
\begin{aligned}
\frac{\partial \log M E}{\partial \tau_{y}} & =\frac{\partial \log \Sigma_{v p}}{\partial \tau_{y}}-\frac{1}{2} \frac{\partial \log \Sigma_{v}}{\partial \tau_{y}}-\frac{1}{2} \frac{\partial \log \Sigma_{p}}{\partial \tau_{y}} \\
& =\frac{\tau_{a}}{\tau_{f}} \frac{\phi}{\tau_{y}}-\frac{1}{2} \frac{\phi^{2} \tau_{a} \tau_{x}}{\tau_{y}^{2}\left(\tau_{a}+\tau_{x} \tau_{\xi}\right)}+o(1) .
\end{aligned}
$$

Note that $\frac{\phi}{\tau_{y}}=\frac{(\gamma+1)\left(\tau_{a}+\tau_{x}\right)\left(\tau_{f}+2 \tau_{s}+\gamma \tau_{s}\right)}{\tau_{f} \tau_{x}(\gamma+2)\left(\tau_{f}+\tau_{s}+\gamma \tau_{s}\right)}+o(1)$ by (A31). So, if $\tau_{\xi}$ is sufficiently large, $\frac{1}{2} \frac{\phi^{2} \tau_{a} \tau_{x}}{\tau_{y}^{2}\left(\tau_{a}+\tau_{x} \tau_{\xi}\right)} \rightarrow 0$, and thus we have $\frac{\partial \log M E}{\partial \tau_{y}}>0$.

\section{Proof of Proposition 6}

Proof of Part (a). Suppose $\tau_{f} \rightarrow \infty$ and $\tau_{a}<\infty$, so that capital providers do not learn from prices but speculators still trade on information about $\tilde{a}$.

By equation (A12), we have $\phi \rightarrow 0$ as $\tau_{f} \rightarrow \infty$ for any given positive $\tau_{x}$ and $\tau_{y}$. (This makes sense, since speculators no longer rely on $\tilde{y}_{i}$ to form trading decisions, given that they know $\tilde{f}$ perfectly and that $\tilde{y}_{i}$ is a noisy signal of $\tilde{f}$.) So, equation (18) implies $\tau_{p}=\frac{\phi^{2} \tau_{x} \tau_{y} \tau_{\xi}}{\tau_{y}+\phi^{2} \tau_{x}} \rightarrow 0$ as $\tau_{f} \rightarrow \infty$. That is, given capital providers now know $\tilde{f}$ perfectly, they no longer learn from prices to glean information regarding $\tilde{f}$. As a result, $\tau_{x}$ and $\tau_{y}$ do not affect $\tau_{p}$ and real efficiency (recall that $\tau_{x}$ and $\tau_{y}$ affect real efficiency only through $\tau_{p}$ ). 
By equations (A4), (A6) and (A7), we have as $\tau_{f} \rightarrow \infty$,

$$
\begin{aligned}
\Sigma_{p} & =\frac{1}{\tau_{a}}+\left(\frac{\phi^{2}}{\tau_{f}}+\frac{\left(\tau_{y}+\phi^{2} \tau_{x}\right)}{\tau_{x} \tau_{y} \tau_{\xi}}\right) \rightarrow \frac{1}{\tau_{a}}+\frac{1}{\tau_{x} \tau_{\xi}} \\
\Sigma_{v} & \rightarrow \frac{(2+\gamma)^{2}}{\tau_{a}(1+\gamma)^{2}} \\
\Sigma_{v p} & =\frac{\gamma+2}{\gamma+1}\left(\frac{1}{\tau_{a}}+\frac{\phi}{\tau_{f}}\right) \rightarrow \frac{\gamma+2}{\gamma+1} \frac{1}{\tau_{a}} .
\end{aligned}
$$

So, $M E=\frac{\Sigma_{v p}}{\sqrt{\Sigma_{v} \Sigma_{p}}} \rightarrow \sqrt{\frac{\tau_{x} \tau_{\xi}}{\tau_{a}+\tau_{x} \tau_{\xi}}}$. Thus, an increase in $\tau_{x}$ will increase $M E$ and an increase in $\tau_{y}$ has no effect on $M E$.

Proof of Part (b). Suppose $\tau_{a} \rightarrow \infty$ and $\tau_{f}<\infty$. By equation (A12), we have $\phi \rightarrow \infty$ as $\tau_{a} \rightarrow \infty$. (This makes sense: Given that $\tilde{x}_{i}$ is useless when $\tilde{a}$ is perfectly known, speculators' trading strategy depends only on $\tilde{y}_{i}$.) So, for any fixed $\tau_{x}>0$ and $\tau_{y}>0$, we have $\tau_{p}=\frac{\phi^{2} \tau_{x} \tau_{y} \tau_{\xi}}{\tau_{y}+\phi^{2} \tau_{x}} \rightarrow \tau_{y} \tau_{\xi}$, and thus, $\tau_{p}$ and real efficiency are not affected by $\tau_{x}$, and they are positively affected by $\tau_{y}$. Since $\tau_{x}$ and $\tau_{y}$ affect real efficiency only through $\tau_{p}$ and since real efficiency improves with $\tau_{p}$ only when $\gamma$ is small, we know changing $\tau_{x}$ does not affect real efficiency and increasing $\tau_{y}$ improves real efficiency for a sufficiently small $\gamma$.

By equations (A4), (A6) and (A7), as $\tau_{a} \rightarrow \infty$, we have:

$$
\begin{aligned}
\Sigma_{p} & =\frac{1}{\tau_{a}}+\phi^{2}\left(\frac{1}{\tau_{f}}+\frac{1}{\tau_{p}}\right)=\phi^{2}\left(\frac{1}{\tau_{f}}+\frac{1}{\tau_{y} \tau_{\xi}}\right)+o(1) \\
\Sigma_{v} & =\frac{\left((1+\gamma) \tau_{f}+(2+\gamma)\left(\tau_{y} \tau_{\xi}+\tau_{s}+\gamma \tau_{s}\right)\right)^{2}+\tau_{f} \tau_{y} \tau_{\xi}}{\tau_{f}\left((1+\gamma)\left(\tau_{f}+\tau_{y} \tau_{\xi}+\tau_{s}+\gamma \tau_{s}\right)\right)^{2}}+o(1) \\
\Sigma_{v p} & =\frac{\gamma+2}{\gamma+1} \frac{\phi}{\tau_{f}}+o(1)
\end{aligned}
$$

So, $M E=\frac{\Sigma_{v p}}{\sqrt{\Sigma_{v} \Sigma_{p}}}=\frac{\frac{\gamma+2}{\gamma+1} \frac{1}{\tau_{f}}}{\sqrt{\left(\frac{\left((1+\gamma) \tau_{f}+(2+\gamma)\left(\tau_{y} \tau_{\xi}+\tau_{s}+\gamma \tau_{s}\right)\right)^{2}+\tau_{f} \tau_{y} \tau_{\xi}}{\tau_{f}\left((1+\gamma)\left(\tau_{f}+\tau_{y} \tau_{\xi}+\tau_{s}+\gamma \tau_{s}\right)\right)^{2}}\right)\left(\frac{1}{\tau_{f}}+\frac{1}{\tau_{y} \tau_{\xi}}\right)}}+o(1)$.

Thus, as $\tau_{a} \rightarrow \infty, \tau_{x}$ does not affect $M E$. Direct computation shows

$$
\frac{\partial\left(\frac{\left((1+\gamma) \tau_{f}+(2+\gamma)\left(\tau_{y} \tau_{\xi}+\tau_{s}+\gamma \tau_{s}\right)\right)^{2}+\tau_{f} \tau_{y} \tau_{\xi}}{\tau_{f}\left((1+\gamma)\left(\tau_{f}+\tau_{y} \tau_{\xi}+\tau_{s}+\gamma \tau_{s}\right)\right)^{2}}\right)\left(\frac{1}{\tau_{f}}+\frac{1}{\tau_{y} \tau_{\xi}}\right)}{\partial \tau_{y}}<0,
$$

and hence, increasing $\tau_{y}$ increases $M E$. 


\section{References}

Amador, M., and P. O. Weill (2010), "Learning from prices: Public Communication and Welfare," Journal of Political Economy 118, 866-907.

Angeletos, G. M.,and A. Pavan (2004), "Transparency of Information and Coordination in Economies with Investment Complementarities," American Economic Review 94, 91-98.

Angeletos, G. M., and A. Pavan (2007), "Efficient Use of Information and Social Value of Information," Econometrica 75, 1103-1142.

Bai, J., T. Philippon, and A. Savov (2013), "Have Financial Markets Become More Informative?" Working Paper.

Bakke, T. E., and T. M. Whited (2010), "Which Firms Follow the Market? An Analysis of Corporate Investment Decisions," Review of Financial Studies 23, 1941-1980.

Beyer, A., D. Cohen, T. Lys, and B. Walther (2010), "The Financial Reporting Environment: Review of the Recent Literature," Journal of Accounting and Economics 50, 296-343.

Bond, P., A. Edmans, and I. Goldstein (2012), "The Real Effects of Financial Markets," Annual Review of Financial Economics 4, 339-360.

Bond P, and H. Eraslan (2010). "Information-Based Trade," Journal of Economic Theory $145,1675-1703$.

Brown, K., W.V. Harlow, and S. M. Tinic (1988), "Risk Aversion, Uncertain Information, and Market Efficiency," Journal of Financial Economics 22, 355-385.

Chen, Q., I. Goldstein, and W. Jiang (2007), "Price Informativeness and Investment Sensitivity to Stock Price," Review of Financial Studies 20, 619-650.

Colombo, L., G. Femminis, and A. Pavan (2013), "Information Acquisition and Welfare," Working Paper.

Cornand, C., and F. Heinemann (2008), "Optimal Degree of Public Information Dissemination," Economic Journal 118, 718-742.

Dow, J., and G. Gorton (1997), "Stock Market Efficiency and Economic Efficiency: Is There a Connection?" Journal of Finance 52, 1087-1129.

Dow, J. and R. Rahi (2003), "Informed Trading, Investment, and Economic Welfare," Journal of Business 76, 430-454.

Edmans, A. (2009), "Blockholder Trading, Market Efficiency, and Managerial Myopia," Journal of Finance 64, 2481-2513. 
Edmans, A., I. Goldstein, and W. Jiang (2012), "The Real Effects of Financial Markets: The Impact of Prices on Takeovers," Journal of Finance 67, 933-971.

Edmans, A., M. Heinle, and C. Huang (2013), "The Real Costs of Disclosure," Wharton Working Paper.

Fama, E. F. (1970), "Efficient Capital Markets: A Review of Theory and Empirical Work," Journal of Finance 25, 383-417.

Fama E.F. and M. Miller (1972), The Theory of Finance. Holt, Rinehart \& Winston, New York.

Foucault, T., and L. Frésard (2013), "Learning from Peers' Stock Prices and Corporate Investment," forthcoming in Journal of Financial Economics.

Foucault, T., and T. Gehrig (2008), "Stock Price Informativeness, Cross-Listings, and Investment Decisions," Journal of Financial Economics 88, 146-168.

Froot, K., D. Scharfstein, and J. Stein (1992), "Herd on the Street: Informational Inefficiencies in a Market with Short-Term Speculation," Journal of Finance 47, 1461-1484.

Gao, P., and P. J. Liang (2013), "Informational Feedback, Adverse Selection, and Optimal Disclosure Policy," Journal of Accounting Research 51, 1133-1158.

Goldman, E. (2005), "Organizational Form, Information Collection, and the Value of the Firm," Journal of Business 78, 817-839.

Goldman E, and G. Strobl (2013), "Large Shareholder Trading and the Complexity of Corporate Investments," Journal of Financial Intermediation 22, 106-122.

Goldstein, I., and A. Guembel (2008), "Manipulation and the Allocational Role of Prices," Review of Economic Studies 75, 133-164.

Goldstein, I., and L. Yang (2012), "Information Diversity and Market Efficiency Spirals," Working Paper.

Goldstein, I., E. Ozdenoren, and K. Yuan (2013), "Trading Frenzies and Their Impact on Real Investment," forthcoming in Journal of Financial Economics.

Grossman, S., and J. Stiglitz (1980), "On the Impossibility of Informationally Efficient Markets," American Economic Review 70, 393-408.

Hayek F. (1945), "The Use of Knowledge in Society," American Economic Review 35, $519-530$.

Hellwig, C. (2005), "Heterogeneous Information and the Welfare Effects of Public Information Disclosures," Working Paper.

Holmstrom, B., and J. Tirole (1993), "Market Liquidity and Performance Monitoring," Journal of Political Economy 101, 678-709. 
Kondor, P. (2012), "The More We Know about the Fundamental, the Less We Agree on the Price," Review of Economic Studies 79), 1175-1207.

Kyle, A. S. (1985), "Continuous Auctions and Insider Trading," Econometrica 53, 13151335.

Liu, Z., P. Wang, and T. Zha (2013), "Land-Price Dynamics and Macroeconomic Fluctuations," Econometrica 81, 1147-1184.

Luo, Y. (2005), "Do Insiders Learn from Outsiders? Evidence From Mergers and Acquisitions," Journal of Finance 60, 1951-1982.

Morris, S., and H. S. Shin (2002), "The Social Value of Public Information," American Economic Review 92, 1521-1534.

O’Hara, M. (1997). Market Microstructure Theory. Blackwell Publishers.

Ozdenoren, E., and K. Yuan (2008), "Feedback Effects and Asset Prices," Journal of Finance $63,1939-1975$.

Ozsoylev, H., and J. Walden (2011), "Asset Pricing in Large Information Networks," Journal of Economic Theory 146, 2252-2280.

Peress, J. (2010), "Product Market Competition, Insider Trading and Stock Market Efficiency," Journal of Finance 65, 1-43.

Sockin, M., and W. Xiong (2013), "Informational Frictions and Commodity Markets," Princeton Working Paper.

Subrahmanyam, A., and S. Titman (2001), "Feedback From Stock Prices to Cash Flows," Journal of Finance 56, 2389-2413.

Subrahmanyam, A., and S. Titman (2013), "Financial Market Shocks and the Macroeconomy," Working Paper.

Veldkamp, L., and J. Wolfers (2007), "Aggregate Shocks or Aggregate Information? Costly Information and Business Cycle Comovement," Journal of Monetary Economics 54(S), 37-55. 


\section{Figure 1: Market Efficiency and Real Efficiency}

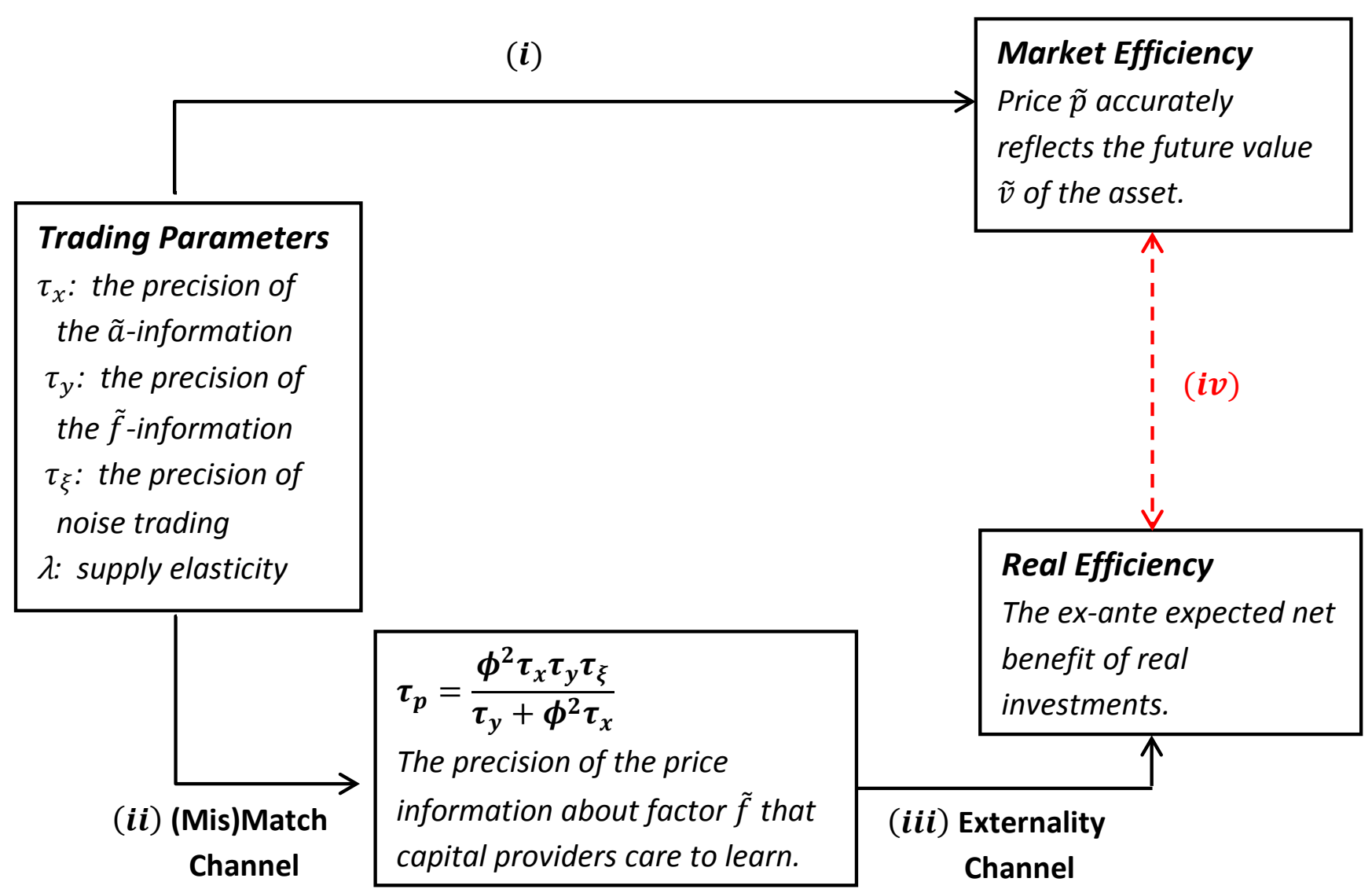

This figure illustrates how trading can affect market efficiency, the information learned by capital providers from the price, and real investment efficiency. 


\section{Figure 2: The Effect of $\tau_{x}$}

(a)

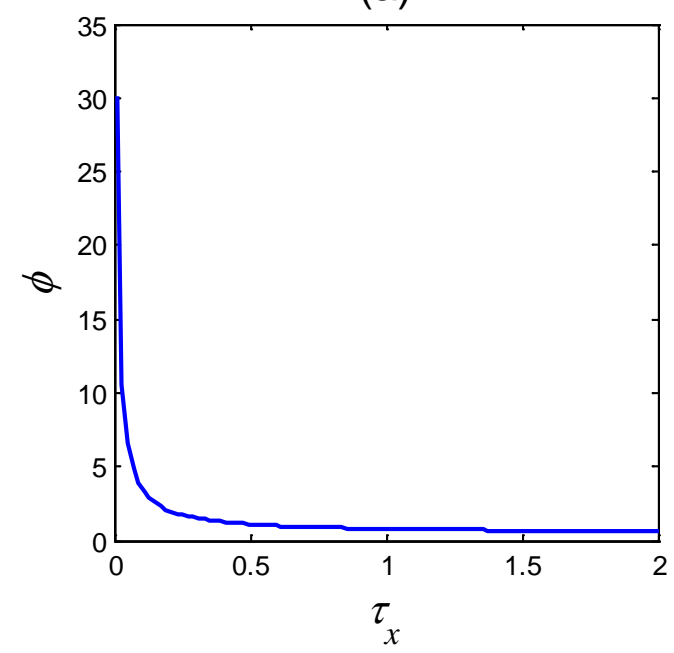

(c)

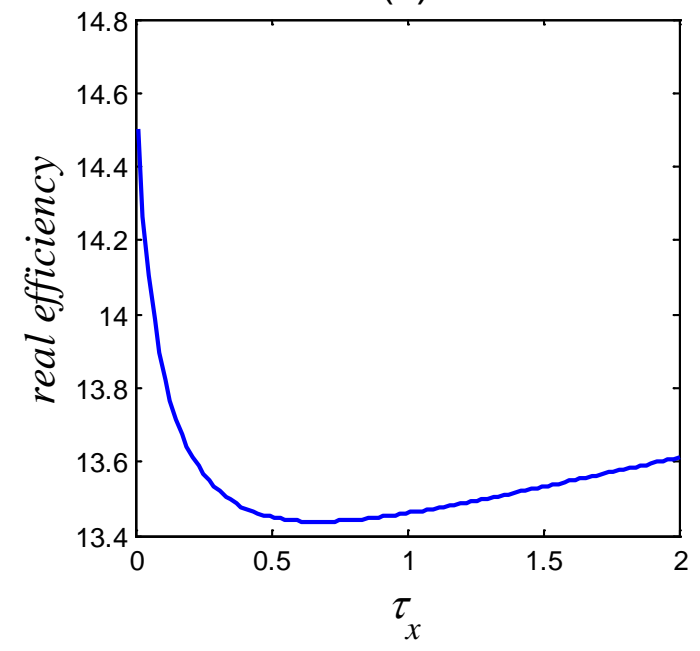

(b)

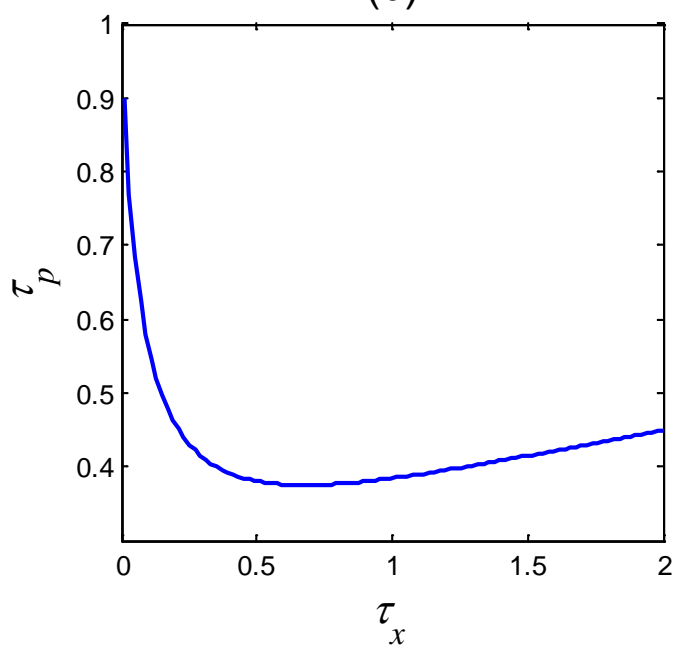

(d)

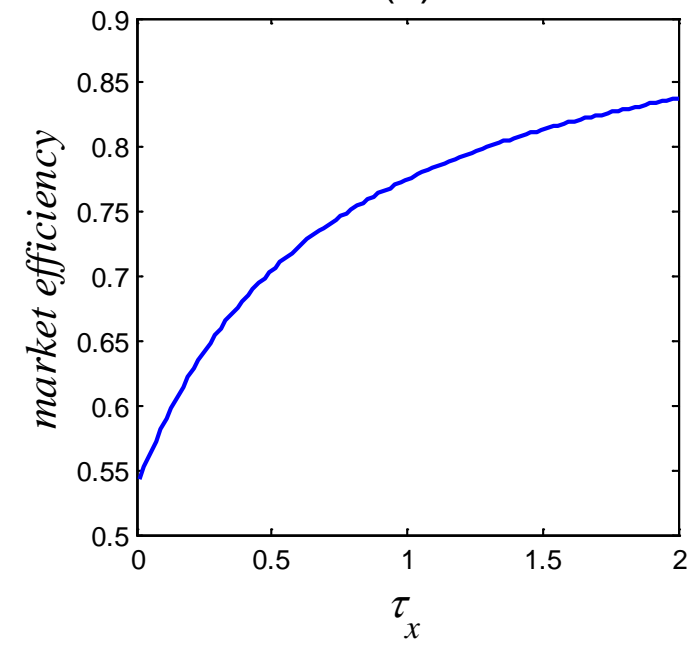

Figure 2 plots the trading and efficiency implications of the precision $\tau_{x}$ of speculators' private information about factor $\tilde{a}$ that capital providers do not care to learn. $\tau_{y}$ is the precision of speculators' private information about factor $\tilde{f}$ that capital providers wish to learn. $\tau_{a}$ and $\tau_{f}$ are the prior precisions of factors $\tilde{a}$ and $\tilde{f} . \tau_{s}$ is the precision of capital providers' private information about factor $\tilde{f} . \tau_{\xi}$ is the precision of noise trading. $\lambda$ is the noisy supply elasticity. $\beta$ is the fraction of output captured by capital providers. $c_{0}$ controls the size of the cost relative to the output. $\gamma$ is a parameter controlling the negative externality in capital providers' cost functions. The parameter values other than $\tau_{y}$ are fixed as follows: $\tau_{a}=\tau_{f}=\tau_{y}=\tau_{s}=\tau_{\xi}=1$, $\beta=1 / 2, c_{0}=1, \gamma=0$, and $\lambda=2$. 
Figure 3: The Effect of $\tau_{y}$

(a)

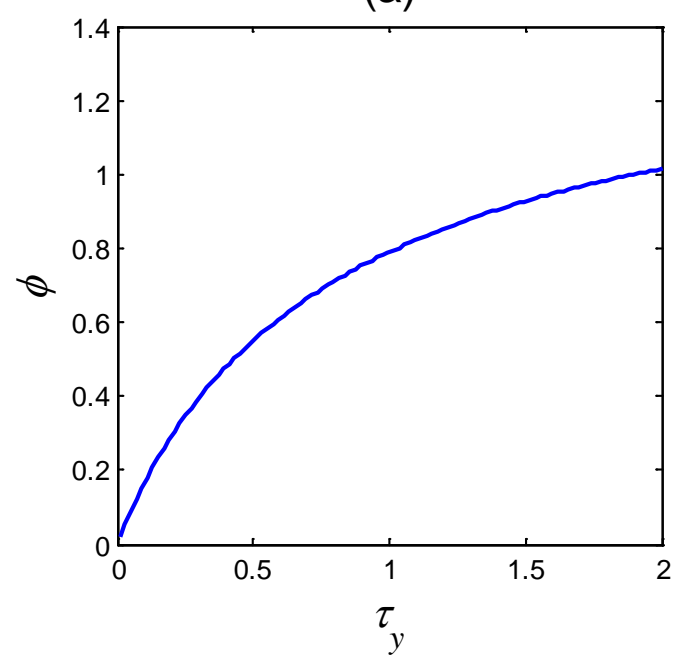

(c)

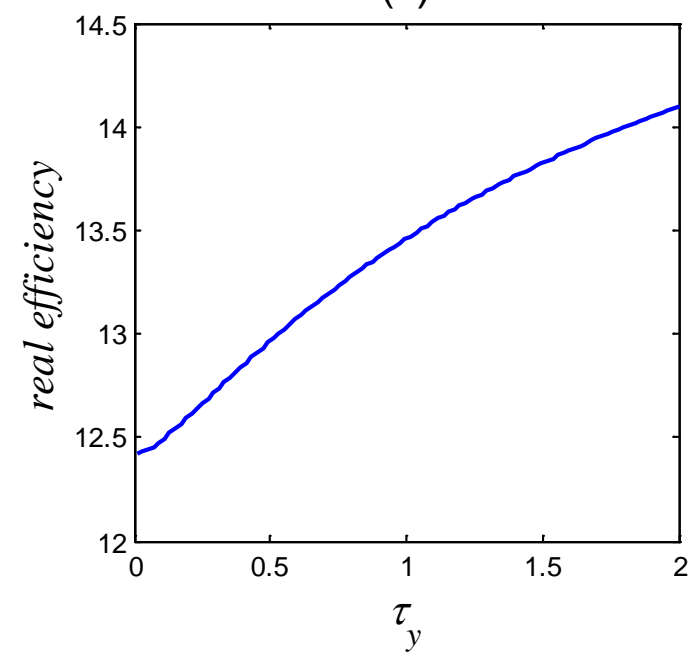

(b)

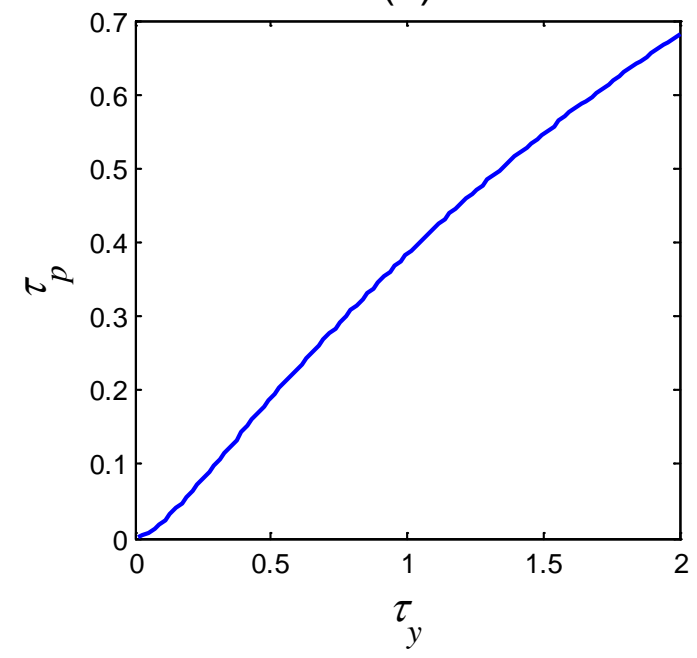

(d)

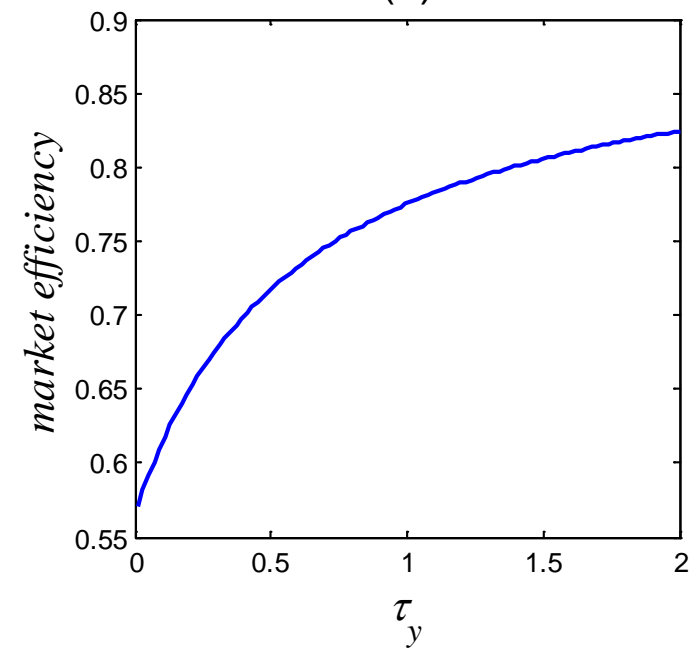

Figure 3 plots the trading and efficiency implications of the precision $\tau_{x}$ of speculators' private information about factor $\tilde{f}$ that capital providers care to learn. $\tau_{x}$ is the precision of speculators' private information about factor $\tilde{a}$ that capital providers know perfectly and do not care to learn. $\tau_{a}$ and $\tau_{f}$ are the prior precisions of factors $\tilde{a}$ and $\tilde{f} . \tau_{s}$ is the precision of capital providers' private information about factor $\tilde{f} . \tau_{\xi}$ is the precision of noise trading. $\lambda$ is the noisy supply elasticity. $\beta$ is the fraction of output captured by capital providers. $c_{0}$ controls the size of the cost relative to the output. $\gamma$ is a parameter controlling the negative externality in capital providers' cost functions. The parameter values other than $\tau_{x}$ are fixed as follows: $\tau_{a}=\tau_{f}=\tau_{x}=\tau_{s}=$ $\tau_{\xi}=1, \beta=1 / 2, c_{0}=1, \gamma=0$, and $\lambda=2$. 


\section{Figure 4: The Effect of $\tau_{x}$ in Extended Economies}

(a1)

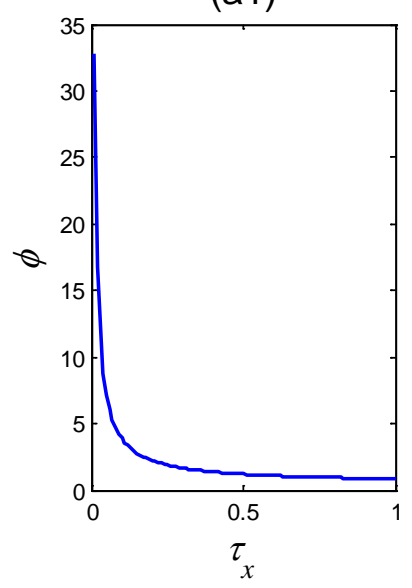

(b1)

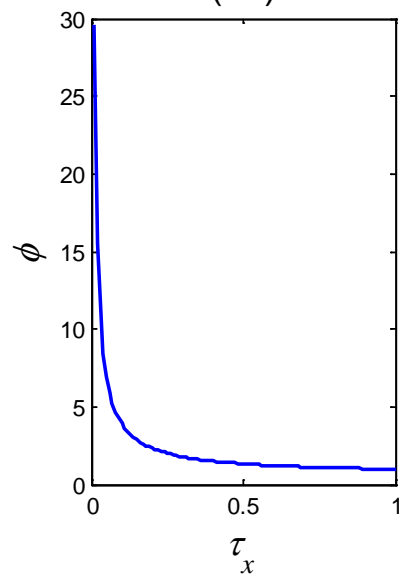

(a2)

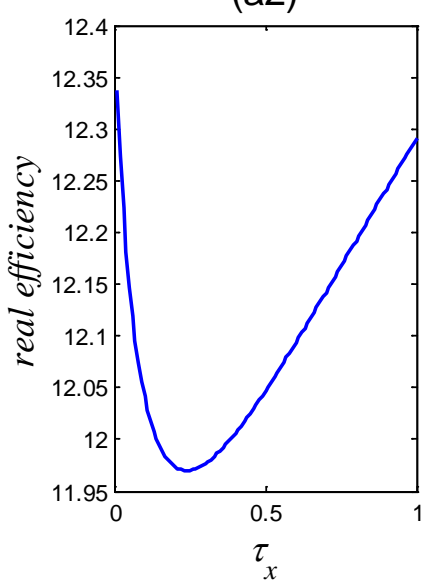

(b2)

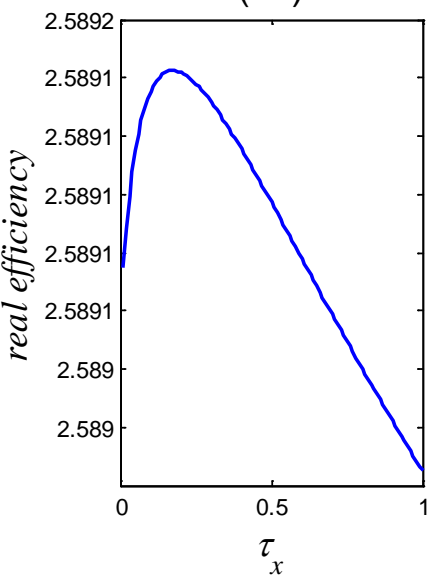

(a3)

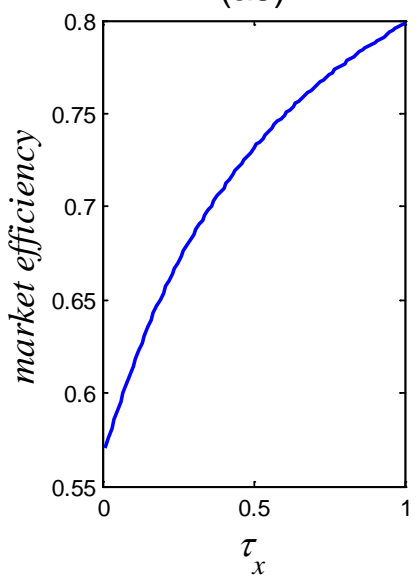

(b3)

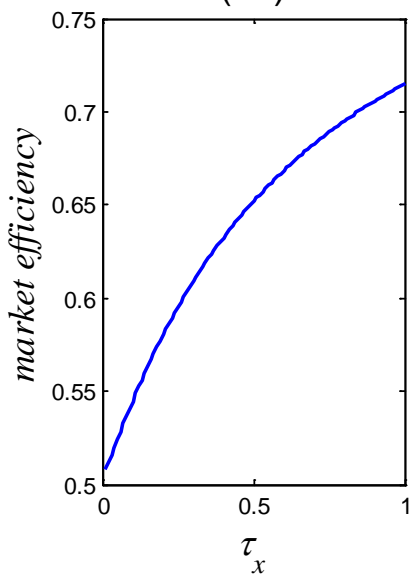

Figure 4 plots the trading and efficiency implications of the precision $\tau_{x}$ of speculators' private information about factor $\tilde{a}$ in the extended economies in which capital provider do not know $\tilde{a}$ perfectly. $\tau_{y}$ is the precision of speculators' private signal about factor $\tilde{f} . \tau_{z}$ and $\tau_{s}$ are the precisions of capital providers' private information about factors $\tilde{a}$ and $\tilde{f}$, respectively. $\tau_{a}$ and $\tau_{f}$ are the prior precisions of factors $\tilde{a}$ and $\tilde{f} . \tau_{\xi}$ is the precision of noise trading. $\lambda$ is the noisy supply elasticity. $\beta$ is the fraction of output captured by capital providers. $c_{0}$ controls the size of the cost relative to the output. $\gamma$ is a parameter controlling the negative externality in capital providers' cost functions. In all panels, we set $\tau_{a}=\tau_{f}=\tau_{y}=\tau_{s}=\tau_{\xi}=1, \tau_{z}=5, \beta=1 / 2$, $c_{0}=1$, and $\lambda=2$. In the upper panels (a1)-(53), we set $\gamma=0$, and in the lower panels (b1)-(b3), we set $\gamma=5$. 


\section{Figure 5: The Effect of $\tau_{y}$ in Extended Economies}

(a1)

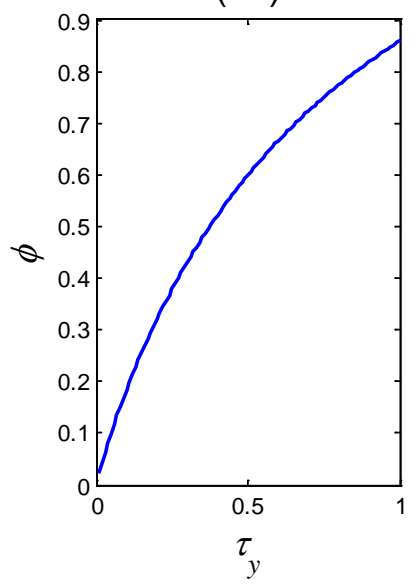

(b1)

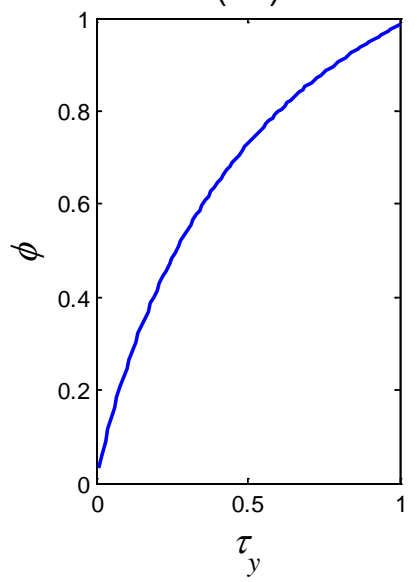

(a2)

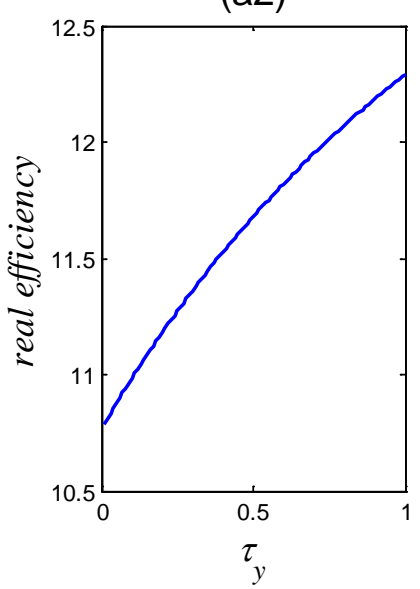

(b2)

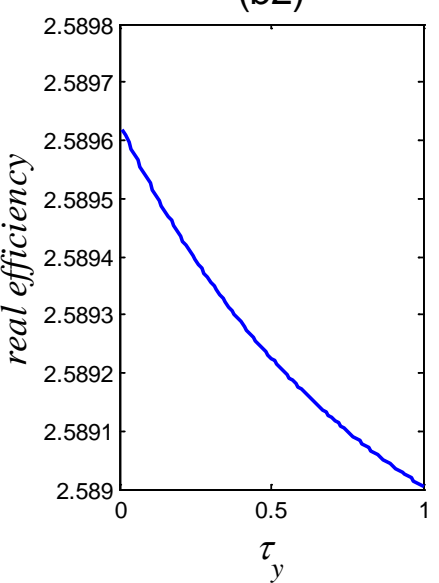

(a3)

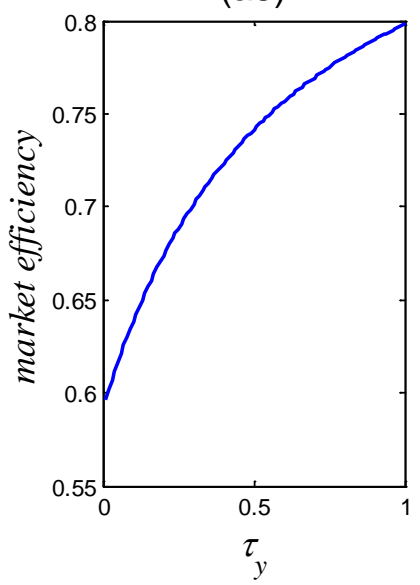

(b3)

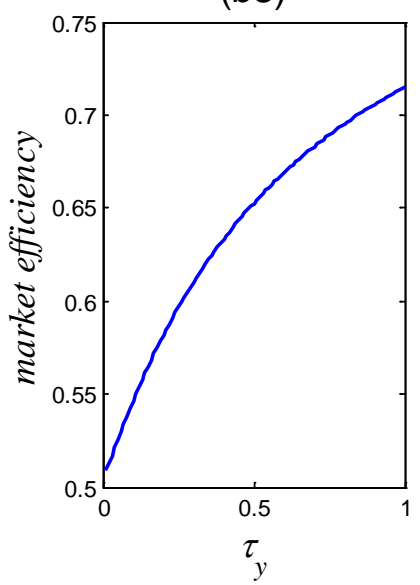

Figure 5 plots the trading and efficiency implications of the precision $\tau_{y}$ of speculators' private information about factor $\tilde{f}$ in the extended economies in which capital provider do not know $\tilde{a}$ perfectly. $\tau_{x}$ is the precision of speculators' private signal about factor $\tilde{a} . \tau_{z}$ and $\tau_{s}$ are the precisions of capital providers' private information about factors $\tilde{a}$ and $\tilde{f}$, respectively. $\tau_{a}$ and $\tau_{f}$ are the prior precisions of factors $\tilde{a}$ and $\tilde{f}$. $\tau_{\xi}$ is the precision of noise trading. $\lambda$ is the noisy supply elasticity. $\beta$ is the fraction of output captured by capital providers. $c_{0}$ controls the size of the cost relative to the output. $\gamma$ is a parameter controlling the negative externality in capital providers' cost functions. In all panels, we set $\tau_{a}=\tau_{f}=\tau_{y}=\tau_{s}=\tau_{\xi}=1, \tau_{z}=5, \beta=1 / 2$, $c_{0}=1$, and $\lambda=2$. In the upper panels (a1)-(53), we set $\gamma=0$, and in the lower panels (b1)-(b3), we set $\gamma=5$. 